\title{
Fractional quantum Hall effect in optical lattices
}

\section{Citation}

Hafezi, M., A. S. Sørensen, E. Demler, and M. D. Lukin. 2007. “Fractional Quantum Hall Effect in Optical Lattices." Physical Review A 76 (2) (August 28). doi:10.1103/physreva.76.023613.

\section{Published Version}

doi:10.1103/PhysRevA.76.023613

\section{Permanent link}

http://nrs.harvard.edu/urn-3:HUL.InstRepos:27890258

\section{Terms of Use}

This article was downloaded from Harvard University's DASH repository, and is made available under the terms and conditions applicable to Other Posted Material, as set forth at http:// nrs.harvard.edu/urn-3:HUL.InstRepos:dash.current.terms-of-use\#LAA

\section{Share Your Story}

The Harvard community has made this article openly available.

Please share how this access benefits you. Submit a story.

\section{Accessibility}




\title{
Fractional quantum Hall effect in optical lattices
}

\author{
M. Hafezi, ${ }^{1, *}$ A. S. Sørensen, ${ }^{2}$ E. Demler, ${ }^{1}$ and M. D. Lukin ${ }^{1}$ \\ ${ }^{1}$ Physics Department, Harvard University, Cambridge, Massachusetts 02138, USA \\ ${ }^{2}$ QUANTOP, Danish National Research Foundation Centre of Quantum Optics, Niels Bohr Institute, \\ University of Copenhagen, DK-2100 Copenhagen Ø, Denmark
}

(Received 6 June 2007; published 28 August 2007)

\begin{abstract}
We analyze a recently proposed method to create fractional quantum Hall (FQH) states of atoms confined in optical lattices [A. Sørensen et al., Phys. Rev. Lett. 94, 086803 (2005)]. Extending the previous work, we investigate conditions under which the $\mathrm{FQH}$ effect can be achieved for bosons on a lattice with an effective magnetic field and finite on-site interaction. Furthermore, we characterize the ground state in such systems by calculating Chern numbers which can provide direct signatures of topological order and explore regimes where the characterization in terms of wave-function overlap fails. We also discuss various issues which are relevant for the practical realization of such $\mathrm{FQH}$ states with ultracold atoms in an optical lattice, including the presence of a long-range dipole interaction which can improve the energy gap and stabilize the ground state. We also investigate a detection technique based on Bragg spectroscopy to probe these systems in an experimental realization.
\end{abstract}

DOI: 10.1103/PhysRevA.76.023613

PACS number(s): 03.75.Lm, 73.43.-f

\section{INTRODUCTION}

With recent advances in the field of ultracold atomic gases, trapped Bose-Einstein condensates (BECs) have become an important system to study many-body physics such as quantum phase transitions. In particular, the ability to dynamically control the lattice structure and the strength of interaction as well as the absence of impurities in BECs confined in optical lattices has led to the recent observation of the superfluid-to-Mott-insulator transition [1-5]. At the same time, there has been a tremendous interest in studying rotating BECs in harmonic traps; at sufficient rotation an Abrikosov lattice of quantized vortices has been observed [6] and realization of strongly correlated quantum states similar to the fractional quantum Hall states has been predicted to occur at higher rotation rates [7-9]. In these proposals, the rotation can play the role of an effective magnetic field for the neutral atoms, and in analogy with electrons, the atoms may enter into a state described by the Laughlin wave function, which was introduced to describe the fractional quantum Hall effect. While this approach yields a stable ground state separated from all excited states by an energy gap, in practice this gap is rather small because of the weak interactions among the particles in the magnetic traps typically used. In optical lattices, the interaction energies are much larger because the atoms are confined in a much smaller volume, and the realization of the fractional quantum Hall effect in optical lattices could therefore lead to a much higher energy gap and be much more robust. In a recent paper [10], it was shown that it is indeed possible to realize the fractional quantum Hall effect in an optical lattice and that the energy gap achieved in this situation is a fraction of the tunneling energy, which can be considerably larger than the typical energy scales in a magnetic trap.

In addition to being an interesting system in its own right, the fractional quantum Hall effect is also interesting from the

\footnotetext{
*hafezi@fas.harvard.edu
}

point of view of topological quantum computation [11]. In these schemes quantum states with fractional statistics can potentially perform fault-tolerant quantum computation. So far, there has been no direct experimental observation of fractional statistics although some signatures have been observed in electron interferometer experiments $[12,13]$. Strongly correlated quantum gases can be a good alternative where the systems are more controllable and impurities are not present. Therefore, realization of fractional quantum Hall $(\mathrm{FQH})$ states in atomic gases can be a promising resource for topological quantum computations in the future.

As noted above the FQH effect can be realized by simply rotating and cooling atoms confined in a harmonic trap. In this situation, it can be shown that the Laughlin wave function exactly describes the ground state of the many-body system $[7,14]$. In optical lattices, on the other hand, there are a number of questions which need to be addressed. First of all, it is unclear to what extent the lattice modifies the fractional quantum Hall physics. For a single particle, the lattice modifies the energy levels from being simple Landau levels into the fractal structure known as the Hofstadter butterfly [15]. In the regime where the magnetic flux going through each lattice $\alpha$ is small, one expects that this will be of minor importance and in Ref. [10] it was argued that the fractional quantum Hall physics persists until $\alpha \leqq 0.3$. In this paper, we extend and quantify predictions carried out in Ref. [10]. Whereas Ref. [10] only considered the effect of an infinite on-site interaction, we extend the analysis to finite interactions. Furthermore, where Ref. [10] mainly argued that the ground state of the atoms was in a quantum Hall state by considering the overlap of the ground state found by numerical diagonalization with the Laughlin wave function, we provide further evidence for this claim by characterizing the topological order of the system by calculating Chern numbers. These calculations thus characterize the order in the system even for parameter regimes where the overlap with the Laughlin wave function is decreased by the lattice structure. 
In addition to considering these fundamental features of the FQH states on a lattice, which are applicable regardless of the system being used to realize the effect, we also study a number of questions which are of particular interest to experimental efforts toward realizing the effect with atoms in optical lattices. In particular, we show that adding dipole interactions between the atoms can be used to increase the energy gap and stabilize the ground state. Furthermore, we study Bragg spectroscopy of atoms in the lattice and show that this is a viable method to identify the quantum Hall states created in an experiment, and we discuss a method to generate an effective magnetic field for the neutral atoms in the lattice.

The paper is organized as follows: In Sec. II, we study the system with finite on-site interaction. In Sec. III we introduce Chern numbers to characterize the topological order of the system. The effect of the dipole-dipole interaction is elaborated on in Sec. IV A. Section IV B studies the case of $\nu$ $=1 / 4$ filling factor. Section V is dedicated to explore Bragg spectroscopy of the system. Section VI outlines an approach for generating the type of the Hamiltonian studied in this paper.

\section{QUANTUM HALL STATE OF BOSONS ON A LATTICE}

\section{A. Model}

The fractional quantum Hall effect occurs for electrons confined in a two-dimensional (2D) plane under the presence of a perpendicular strong magnetic field. If $N$ is the number of electrons in the system and $N_{\phi}$ is the number of magnetic fluxes measured in units of the quantum magnetic flux $\Phi_{0}$ $=h / e$, then depending on the filling factor $\nu=N / N_{\phi}$ the ground state of the system can form highly entangled states exhibiting rich behaviors, such as incompressibility, charge density waves, and anionic excitations with fractional statistics. In particular, when $\nu=1 / m$, where $m$ is an integer, the ground state of the system is an incompressible quantum liquid which is protected by an energy gap from all other states and in the Landau gauge is well described by the Laughlin wave function [16]

$$
\Psi\left(z_{1}, z_{2}, \ldots, z_{N}\right)=\prod_{j>k}^{N}\left(z_{j}-z_{k}\right)^{m} \prod_{j=1}^{N} e^{-y_{i}^{2} / 2},
$$

where the integer $m$ should be odd in order to meet the antisymmetrization requirement for fermions.

Although the fractional quantum Hall effect occurs for fermions (electrons), bosonic systems with repulsive interactions can exhibit similar behaviors. In particular, the Laughlin states with even $m$ correspond to bosons. In this article, we study bosons since the experimental implementation is more advanced for the ultracold bosonic systems. We study a system of atoms confined in a 2D lattice which can be described by the Bose-Hubbard model [17] with Peierls substitution $[15,18]$,

$$
\begin{aligned}
H= & -J \sum_{x, y} \hat{a}_{x+1, y}^{\dagger} \hat{a}_{x, y} e^{-i \pi \alpha y}+\hat{a}_{x, y+1}^{\dagger} \hat{a}_{x, y} e^{i \pi \alpha x}+\text { H.c. } \\
& +U \sum_{x, y} \hat{n}_{x, y}\left(\hat{n}_{x, y}-1\right),
\end{aligned}
$$

where $J$ is the hopping energy between two neighboring sites, $U$ is the on-site interaction energy, and $2 \pi \alpha$ is the phase acquired by a particle going around a plaquette. This Hamiltonian is equivalent to the Hamiltonian of a $\mathrm{U}(1)$ gauge field (transverse magnetic field) on a square lattice. More precisely, the noninteracting part can be written as

$$
-J \sum_{\langle i j\rangle} a_{i}^{\dagger} a_{j} \exp \left(\frac{2 \pi i}{\Phi_{0}} \int_{i}^{j} \vec{A} \cdot \overrightarrow{d l}\right),
$$

where $\vec{A}$ is the vector potential for a uniform magnetic field and the path of the integral is chosen to be a straight line between two neighboring sites. In the symmetric gauge, the vector potential is written as $\vec{A}=\frac{B}{2}(-y, x, 0)$. Hence, $\alpha$ will be the amount of magnetic flux going through one plaquette.

While the Hamiltonian in Eq. (2) occurs naturally for charged particles in a magnetic field, the realization of a similar Hamiltonian for neutral particles is not straightforward. As we discuss in Sec. VI this may be achieved in a rotating harmonic trap, and this has been very successfully used in a number of experiments in magnetic traps $[19,20]$, but the situation is more complicated for an optical lattice. However, there have been a number of proposals for lattice realization of a magnetic field [10,21,22], and recently it has been realized experimentally [23]. Popp et al. [24] have studied the realization of fractional Hall states for a few particles in individual lattice sites. An approach for rotating the entire optical lattice is discussed in Sec. VI. The essence of the above Hamiltonian is a nonzero phase that a particle should acquire when it goes around a plaquette. This phase can be obtained, for example, by alternating the tunneling and adding a quadratic superlattice potential [10] or by simply rotating the lattice (Sec. VI). The advantage of confining ultracold gases in an optical lattice is to enhance the interaction between atoms, which consequently results in a higherenergy gap compared to harmonic trap proposals (e.g., Ref. [7]). This enhancement in the energy gap of the excitation spectrum can alleviate some of the challenges for experimental realization of the quantum Hall state for ultracold atoms.

\section{B. Energy spectrum and overlap calculations}

In order to approximate a large system, we study the system with periodic boundary conditions-i.e., on a torus, where the topological properties of the system are best manifested.

There are two energy scales for the system: the first is the magnetic tunneling term $J \alpha$, which is related to the cyclotron energy in the continuum limit $\hbar \omega_{c}=4 \pi J \alpha$, and the second is the on-site interaction energy $U$. Experimentally, the ratio between these two energy scales can be varied by varying the lattice potential height $[1,17]$ or by Feshbach resonances $[4,25,26]$. Let us first assume that we are in the continuum limit where $\alpha \ll 1$; i.e., the flux through each plaquette is a 
small fraction of a flux quantum. A determining factor for describing the system is the filling factor $\nu=N / N_{\Phi}$, and in this study we mainly focus on the case of $\nu=1 / 2$, since this will be the most experimentally accessible regime.

We restrict ourselves to the simplest boundary conditions for the single-particle states $t_{s}(\vec{L}) \psi\left(x_{s}, y_{s}\right)=\psi\left(x_{s}, y_{s}\right)$, where $t_{s}(\vec{L})$ is the magnetic translation operator which translates the single-particle states $\psi\left(x_{s}, y_{s}\right)$ around the torus. The definition and detailed discussion of the boundary conditions will be elaborated in Sec. III. The discussed quantities in this section, such as energy spectrum, gap, and overlap, do not depend on the boundary condition angles (this is also verified by our numerical calculation).

In the continuum case, for the filling fraction $\nu=1 / 2$, the Laughlin state in Landau gauge $(\vec{A}=-B y \hat{x})$ is given by Eq. (1) with $m=2$. The generalization of the Laughlin wave function to a torus takes the form [27]

$$
\Psi\left(z_{1}, z_{2}, \ldots, z_{N}\right)=f_{\text {rel }}\left(z_{1}, z_{2}, \ldots, z_{N}\right) F_{\text {c.m. }}(Z) \exp \left(-\sum_{i} y_{i}^{2} / 2\right),
$$

where $f_{\text {rel }}$ is the relative part of the wave function and is invariant under collective shifts of all $z_{i}$ 's by the same amount, and $F_{\text {c.m. }}(Z)$ is related to the motion of the center of mass and is only a function of $Z=\Sigma_{i} z_{i}$. For a system on a torus of the size $\left(L_{x} \times L_{y}\right)$, we write the wave function with the help of $\theta$ functions, which are the proper oscillatory periodic functions and are defined as $\vartheta\left[{ }_{b}^{a}\right](z \mid \tau)$ $=\Sigma_{n} e^{i \pi \tau(n+a)^{2}+2 \pi i(n+a)(z+b)}$ where the sum is over all integers. For the relative part we have

$$
f_{\text {rel }}=\prod_{i<j} \vartheta\left[\begin{array}{c}
\frac{1}{2} \\
\frac{1}{2}
\end{array}\right]\left(\frac{z_{i}-z_{j}}{L_{x}} \mid i \frac{L_{y}}{L_{x}}\right)^{2} .
$$

According to a general symmetry argument by Haldane [28], the center-of-mass wave function $F_{\text {c.m. }}(Z)$ is twofold degenerate for the case of $\nu=1 / 2$ and is given by

$$
F_{\text {c.m. }}(Z)=\vartheta\left[\begin{array}{c}
l / 2+\left(N_{\phi}-2\right) / 4 \\
-\left(N_{\phi}-2\right) / 2
\end{array}\right]\left(\begin{array}{c}
2 \sum_{i} z_{i} \\
L_{x}
\end{array} 2 i \frac{L_{y}}{L_{x}}\right),
$$

where $l=0,1$ refers to the two degenerate ground states. This degeneracy in the continuum limit is due to the translational symmetry of the ground state on the torus, and the same argument can be applied to a lattice system when the magnetic length is much larger than the lattice spacing, $\alpha \ll 1$. For higher magnetic field, the lattice structure becomes more pronounced. However, in our numerical calculation for a moderate magnetic field $\alpha \leq 0.4$, we observe a twofold degeneracy ground state well separated from the excited state by an energy gap. We return to the discussion of the groundstate degeneracy in Sec. III.

In the continuum limit $\alpha \ll 1$, the Laughlin wave function is the exact ground state of the many-body system with a short-range interaction $[7,14,29]$. The reason is that the ground state is composed entirely of states in the lowest Landau level, which minimizes the magnetic part of the Hamiltonian, the first term in Eq. (2). The expectation value of the interacting part of the Hamiltonian-i.e., the second term in Eq. (2) - for the Laughlin state is zero regardless of the strength of the interaction, since it vanishes when the particles are at the same position.

To study the system with a nonvanishing $\alpha$, we have performed a direct numerical diagonalization of the Hamiltonian for a small number of particles. Since we are dealing with identical particles, the states in the Hilbert space can be labeled by specifying the number of particles at each of the lattice sites. In the hard-core limit, only one particle is permitted on each lattice site; therefore, for $N$ particles on a lattice with the number of sites equal to $\left(N_{x}=L_{x} / a, N_{y}\right.$ $=L_{y} / a$ ), where $a$ is the unit lattice side, the Hilbert space size is given by the combination

$$
\left(\begin{array}{c}
N_{x} N_{y} \\
N
\end{array}\right)=\frac{N_{x} N_{y} !}{N !\left(N_{x} N_{y}-N\right) !} .
$$

On the other hand, in case of finite on-site interaction, the particles can be on top of each other, so the Hilbert space is larger and is given by the combination

$$
\left(\begin{array}{c}
N+N_{x} N_{y}-1 \\
N
\end{array}\right) .
$$

In our simulations the dimension of the Hilbert can be raised up to $\sim 4 \times 10^{6}$ and the Hamiltonian is constructed in configuration space by taking into account the tunneling and interacting terms. The tunneling term is written in the symmetric gauge, and we make sure that the phase acquired around a plaquette is equal to $2 \pi \alpha$ and that the generalized magnetic boundary condition is satisfied when the particles tunnel over the edge of the lattice [to be discussed in Sec. III; cf. Eq. (11)]. By diagonalizing the Hamilton, we find the twofold-degenerate ground-state energy which is separated by an energy gap from the excited states and the corresponding wave function in configuration space. The Laughlin wave function (4) can also be written in configuration space by simply evaluating the Laughlin wave function at discrete points, and therefore we can compared the overlap of these two-dimensional subspaces.

\section{Results with the finite on-site interaction}

The energy gap above the ground state and the groundstate overlap with the Laughlin wave function for the case of $\nu=1 / 2$ in a dilute lattice $\alpha \lesssim 0.2$ are depicted in Fig. 1. The Laughlin wave function remains a good description of the ground state even if the strength of the repulsive interaction tends to zero [Fig. 1(a)]. Below, we discuss different limits.

First, we consider $U>0, U \gg J \alpha$ : If the interaction energy scale $U$ is much larger than the magnetic one $(J \alpha)$, all lowenergy states lie in a manifold, where the highest occupation number for each site is one; i.e., this corresponds to the hardcore limit. The ground state is the Laughlin state, and the excited states are various mixtures of Landau states. The ground state is twofold degenerate [28], and the gap reaches 

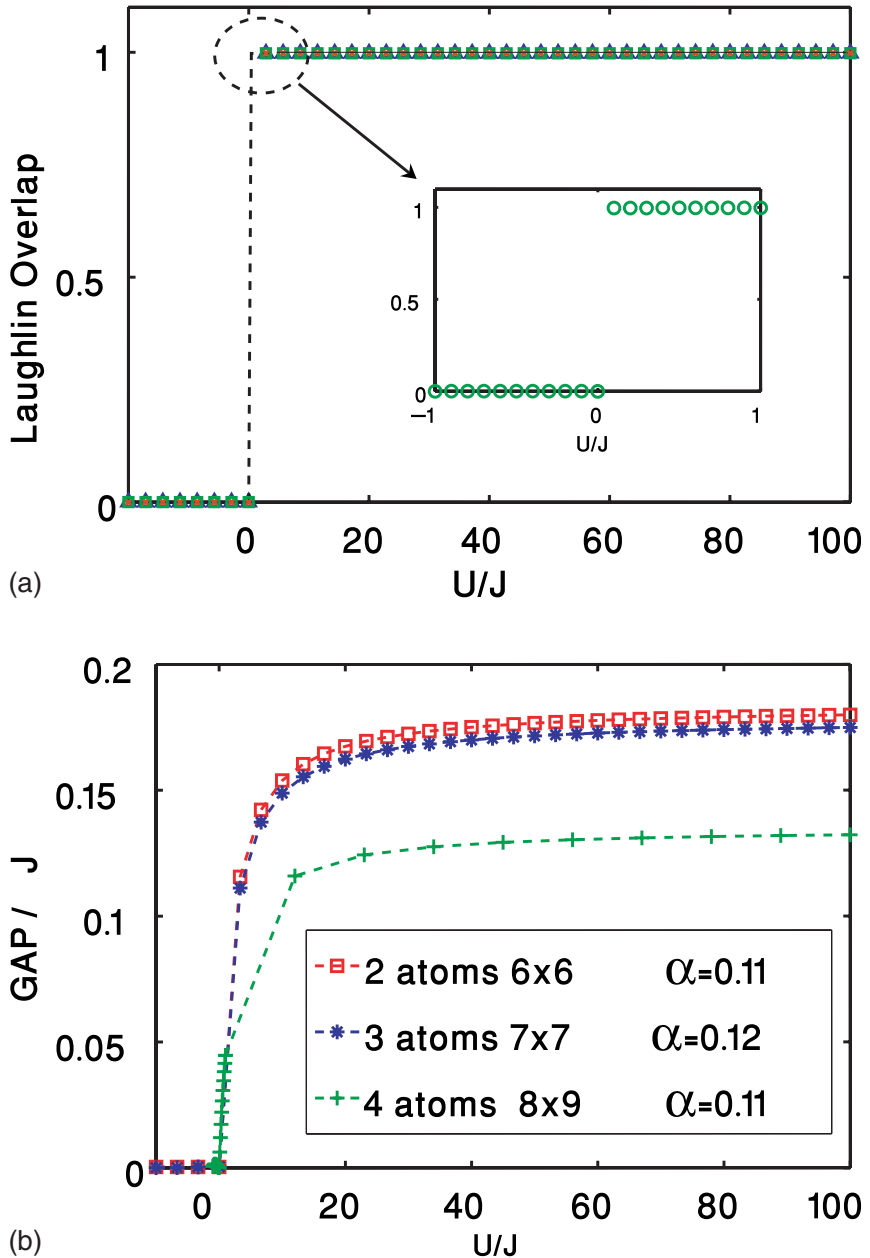

FIG. 1. (Color online) (a) The overlap of the ground state with the Laughlin wave function. For small $\alpha$ the Laughlin wave function is a good description of the ground state for positive interaction strengths. The inset shows the same result of small $U$. (b) The energy gap for $N / N_{\phi}=1 / 2$ as a function of interaction $U / J$ from attractive to repulsive. For a fixed $\alpha$, the behavior does not depend on the number of atoms. The inset defines the particle numbers, lattice sizes, and symbols for both parts (a) and (b).

the value for the hard-core limit at large $U \gtrsim 3 J / \alpha$, as shown in Fig. 2(a). In this limit the gap only depends on the tunneling $J$ and flux $\alpha$, and the gap is a fraction of $J$. These results are consistent with the previous work in Ref. [10].

Second, we consider $|U| \ll J \alpha$. In this regime, the magnetic energy scale $(J \alpha)$ is much larger than the interaction energy scale $U$. For the repulsive regime $(U>0)$, the ground state is the Laughlin state and the gap increases linearly with $\alpha U$, as shown in Fig. 2(b).

Third, we study $U=0$ where the interaction is absent and the ground state becomes highly degenerate. For a single particle on a lattice, the spectrum is the famous Hofstadter's butterfly [15], while in the continuum limit $\alpha \ll 1$, the ground state is the lowest Landau level (LLL). The single-particle degeneracy of the LLL is the number of fluxes going through the surface, $N_{\phi}$. So in the case of $N$ bosons, the lowest energy is obtained by putting $N$ bosons in $N_{\phi}$ levels. Therefore, the many-body ground state's degeneracy should be

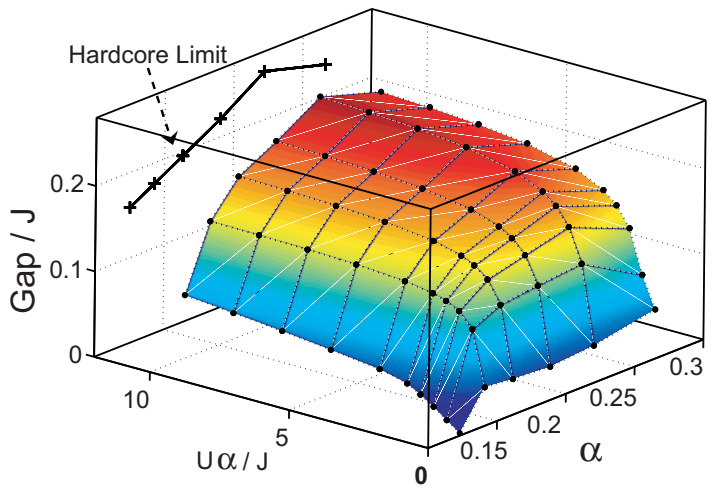

(a)

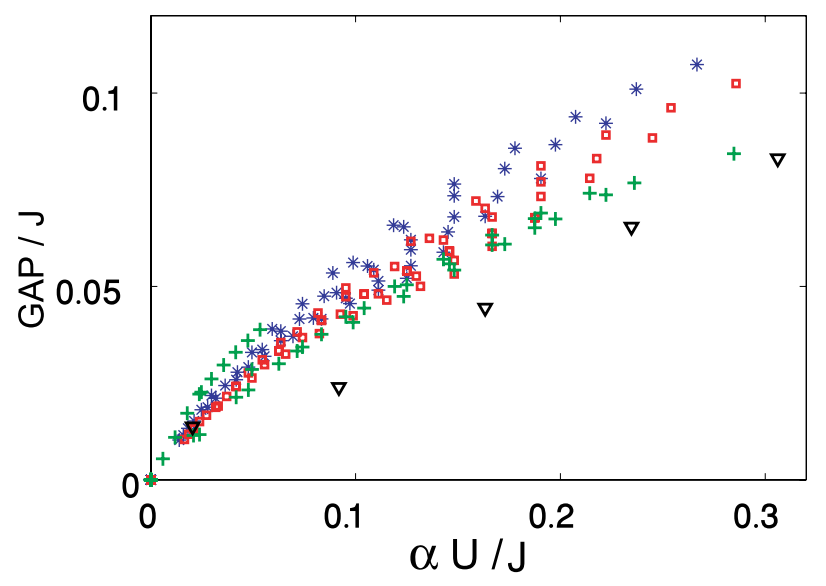

(b)

FIG. 2. (Color online) (a) The energy gap as a function of $\alpha U$ and $\alpha$ for a fixed number of atoms $(N=4)$. The gap is calculated for the parameters marked with dots, and the surface is an extrapolation between the points. (b) Linear scaling of the energy gap with $\alpha U$ for $U \ll J, \alpha \lesssim 0.2$. The results are shown for $N=2(\square), N=3(*)$, $N=4(+)$, and $N=5(\nabla)$. The gap disappears for noninteraction system, and increases with increasing interaction strength $(\propto \alpha U)$ and eventually saturates to the value in the hard-core limit.

$$
\left(\begin{array}{c}
N+N_{\phi}-1 \\
N_{\phi}-1
\end{array}\right)
$$

For example, 3 bosons and 6 fluxes give a 336-fold degeneracy in the noninteracting ground state.

If we increase the amount of phase (flux) per plaquette $(\alpha)$, we are no longer in the continuum limit. The Landaulevel degeneracy will be replaced by $\frac{L_{1} L_{2}}{s}$ where $\alpha=r / s$ is the amount of flux per plaquette and $r$ and $s$ are coprime [30]. Then, the many-body degeneracy will be

$$
\left(\begin{array}{c}
N+\frac{L_{1} L_{2}}{s}-1 \\
\frac{L_{1} L_{2}}{s}-1
\end{array}\right) .
$$


Fourth, we consider $U<0, U \gg J \alpha$ : when $U$ is negative (i.e., attractive interaction) in the limit of the strong interaction regime, the ground state of the system will become a pillar state. In a pillar state, all bosons in the system condensate into a single site. Therefore, the degeneracy of the ground state is $N_{x} \times N_{y}$ and the ground-state manifold can be spanned as

$$
\underset{i}{\oplus} \frac{1}{\sqrt{N !}}\left(a^{\dagger}\right)_{i}^{N}|\mathrm{vac}\rangle
$$

These states will be very fragile and susceptible to collapse [31].

In a lattice, it is also possible to realize the fractional quantum Hall states for attractive interaction in the limit when $|U| \gg J \alpha$. Assume that the occupation number of each site is either 0 or 1 . Since the attraction energy $U$ is very high and there is no channel into which the system can dissipate its energy, the probability for a boson to hop to a site where there is already a boson is infinitesimally small. Therefore the high-energy attraction will induce an effective hard-core constraint in the case of an ultracold system. The energy of these states should be exactly equal to their hard-core ground-state counterparts, since the interaction expectation value of the interaction energy is zero for the Laughlin state. The numerical simulation shows that these two degenerate states indeed have a good overlap with the Laughlin wave function similar to their repulsive hard-core counterparts and also their energies are equal to the hard-core ground state. These states are very similar to repulsively bound atom pairs in an optical lattice which have recently been experimentally observed [4].

So far we have mainly considered a dilute lattice $\alpha$ $\lessgtr 0.2$, where the difference between a lattice and the continuum is very limited. We shall now begin to investigate what happens for larger values of $\alpha$, where the effect of the lattice plays a significant role. Figure 3 shows the groundstate overlap with the Laughlin wave function as a function of the strength of magnetic flux $\alpha$ and the strength of the on-site interaction $U$. As $\alpha$ increases the Laughlin overlap is no longer an exact description of the system since the lattice behavior of the system is more pronounced comparing to the continuum case. This behavior does not depend significantly on the number of particles for the limited number of particles that we have investigated, $N \leqslant 5$. We have, however, not made any modification to the Laughlin wave function to take into account the underlying lattice, and from the calculations presented so far, it is unclear whether the decreasing wavefunction overlap represents a change in the nature of the ground state or whether it is just caused by a modification to the Laughlin wave function due to the difference between the continuum and the lattice. To investigate this, in the next section, we provide a better characterization of the ground state in terms of Chern numbers, which shows that the same topological order is still present in the system for higher values of $\alpha$.

As a summary, we observe that the Laughlin wave function is a good description for the case of a dilute lattice $(\alpha$ $\ll 1$ ), regardless of the strength of the on-site interaction.
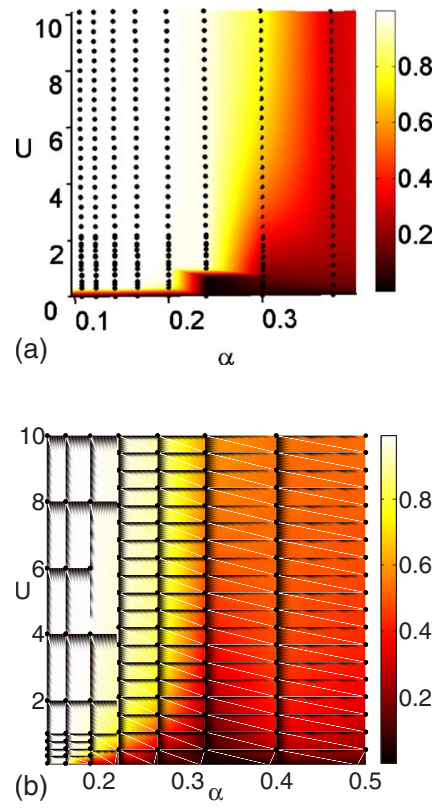

FIG. 3. (Color online) Ground-state overlap with the Laughlin wave function. (a) and (b) are for three and four atoms on a lattice, respectively. $\alpha$ is varied by changing the size of the lattice (the size in the two orthogonal directions differ at most by unity). The Laughlin state ceases to be a good description of the system as the lattice nature of the system becomes more apparent, $\alpha \gtrsim 0.25$. The overlap is only calculated at the positions shown with dots, and the color coding is an extrapolation between the points.

However, the protective gap of the ground state becomes smaller for weaker values of the interaction and in the perturbative regime $U \ll J$ is proportional to $\alpha U$ for $\alpha \lesssim 0.2$.

\section{CHERN NUMBER AND TOPOLOGICAL INVARIANCE}

\section{A. Chern number as a probe of topological order}

In the theory of the quantum Hall effect, it is well understood that the conductance quantization is due to the existence of certain topological invariants, so-called Chern numbers. The topological invariance was introduced by Avron et al. [32] in the context of the Thouless-KohmotoNightingale-den Nijs TKNdN original theory [33] about quantization of the conductance. TKNdN in their seminal work showed that the Hall conductance calculated from the Kubo formula can be expressed as an integral over the magnetic Brillouin zone, which shows the quantization explicitly. The original paper of TKNdN deals with the single-particle problem and Bloch waves which cannot be generalized to topological invariance. The generalization to many-body systems has been done by Niu et al. [34] and also Tao and Haldane [35], by manipulating the phases describing the closed boundary conditions on a torus (i.e., twist angles), for both the integer and fractional Hall systems. These twist angles come from natural generalization of the closed boundary condition.

To clarify the origin of these phases, we start with a single-particle picture. A single particle with charge $(q)$ on a 
torus of the size $\left(L_{x}, L_{y}\right)$ in the presence of a magnetic field $B$ perpendicular to the torus surface is described by the Hamiltonian

$$
H_{s}=\frac{1}{2 m}\left[\left(-i \hbar \frac{\partial}{\partial x}-q A_{x}\right)^{2}+\left(-i \hbar \frac{\partial}{\partial y}-q A_{y}\right)^{2}\right],
$$

where $\vec{A}$ is the corresponding vector potential $\left(\frac{\partial A_{y}}{\partial x}-\frac{\partial A_{x}}{\partial y}=B\right)$. This Hamiltonian is invariant under the magnetic translation

$$
t_{s}(\mathbf{a})=e^{i \mathbf{a} \cdot \mathbf{k}^{s} / \hbar},
$$

where $\mathbf{a}$ is a vector in the plane and $\mathbf{k}^{s}$ is the pseudomomentum, defined by

$$
\begin{aligned}
& k_{x}^{s}=-i \hbar \frac{\partial}{\partial x}-q A_{x}-q B y, \\
& k_{y}^{s}=-i \hbar \frac{\partial}{\partial y}-q A_{y}+q B x .
\end{aligned}
$$

The generalized boundary condition on a torus is given by the single-particle translation

$$
\begin{aligned}
& t_{s}\left(L_{x} \hat{x}\right) \psi\left(x_{s}, y_{s}\right)=e^{i \theta_{1}} \psi\left(x_{s}, y_{s}\right), \\
& t_{s}\left(L_{y} \hat{y}\right) \psi\left(x_{s}, y_{s}\right)=e^{i \theta_{2}} \psi\left(x_{s}, y_{s}\right),
\end{aligned}
$$

where $\theta_{1}$ and $\theta_{2}$ are twist angles of the boundary. The origin of these phases can be understood by noting that the periodic boundary conditions correspond to the torus in Fig. 4(a). The magnetic flux through the surface arises from the field perpendicular to the surface of the torus. However, in addition to this flux, there may also be fluxes due to a magnetic field residing inside the torus or passing through the torus hole, and it is these extra fluxes which give rise to the phases. The extra free angles are all the same for all particles and all states in the Hilbert space, and their time derivative can be related to the voltage drops across the Hall device in two dimensions.

The eigenstates of the Hamiltonian, including the ground state, will be a function of these boundary angles $\Psi^{(\alpha)}\left(\theta_{1}, \theta_{2}\right)$. By defining some integral form of this eigenstate, one can introduce quantities that do not depend on the details of the system, but reveal general topological features of the eigenstates.

First we discuss the simplest situation, where the ground state is nondegenerate, and later we shall generalize this to our situation with a degenerate ground state. The Chern number is in the context of quantum Hall systems related to a measurable physical quantity, the Hall conductance. The boundary-averaged Hall conductance for the (nondegenerate) $\alpha$ th many-body eigenstate of the Hamiltonian is [34,35] $\sigma_{H}^{\alpha}$ $=C(\alpha) e^{2} / h$, where the Chern number $C(\alpha)$ is given by

$$
C(\alpha)=\frac{1}{2 \pi} \int_{0}^{2 \pi} d \theta_{1} \int_{0}^{2 \pi} d \theta_{2}\left(\partial_{1} \mathcal{A}_{2}^{(\alpha)}-\partial_{2} \mathcal{A}_{1}^{(\alpha)}\right),
$$

where $\mathcal{A}_{j}^{(\alpha)}\left(\theta_{1}, \theta_{2}\right)$ is defined as a vector field based on the eigenstate $\Psi^{(\alpha)}\left(\theta_{1}, \theta_{2}\right)$ on the boundary torus $S_{1} \times S_{1}$ by
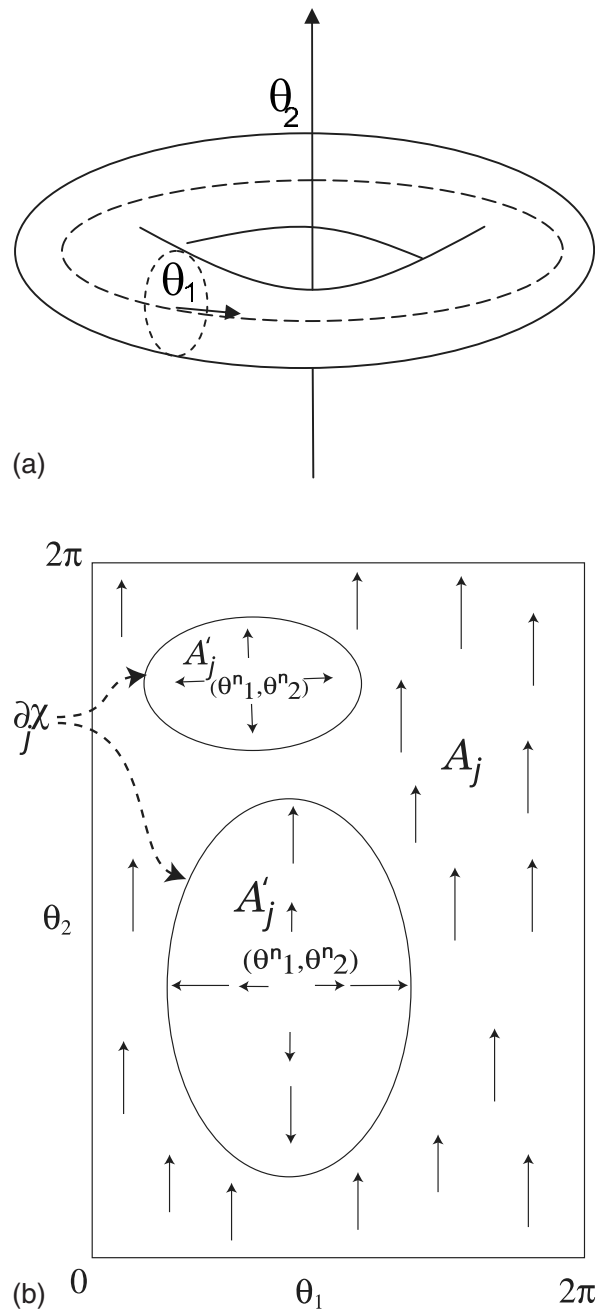

FIG. 4. (a) Twist angles of the toroidal boundary condition. In addition to the flux going through the surface there may also be a flux inside the torus or going through the hole in the middle. When encircling these fluxes the wave function acquires an extra phase represented by the boundary conditions in Eq. (11). (b) Redefining the vector potential around the singularities: $\mathcal{A}_{j}$ is not well defined everywhere on the torus of the boundary condition. Therefore, another vector field $\mathcal{A}^{\prime}{ }_{j}$ with different definition should be introduced around each singularity $\left(\theta_{1}^{n}, \theta_{2}^{n}\right)$ of $\mathcal{A}_{j}$. $\mathcal{A}_{j}$ and $\mathcal{A}_{j}^{\prime}$ are related to each other by a gauge transformation $\chi$, and the Chern number depends only on the loop integrals of $\chi$ around those singularities regions; cf. Eq. (16).

$$
\mathcal{A}_{j}^{(\alpha)}\left(\theta_{1}, \theta_{2}\right) \doteq i\left\langle\Psi^{(\alpha)}\left|\frac{\partial}{\partial \theta_{j}}\right| \Psi^{(\alpha)}\right\rangle .
$$

It should be noted that the wave function $\Psi^{(\alpha)}\left(\theta_{1}, \theta_{2}\right)$ is defined up to a phase factor on the boundary-phase space. Therefore, $\Psi^{(\alpha)}\left(\theta_{1}, \theta_{2}\right)$ and $e^{i f\left(\theta_{1}, \theta_{2}\right)} \Psi^{(\alpha)}\left(\theta_{1}, \theta_{2}\right)$ are physically equivalent for any smooth function $f\left(\theta_{1}, \theta_{2}\right)$. Under this phase change, $\mathcal{A}_{j}^{(\alpha)}\left(\theta_{1}, \theta_{2}\right)$ transforms like a gauge:

$$
\begin{gathered}
\mathcal{A}_{j}^{(\alpha)}\left(\theta_{1}, \theta_{2}\right) \rightarrow \mathcal{A}_{j}^{(\alpha)}\left(\theta_{1}, \theta_{2}\right)-\partial_{j} f\left(\theta_{1}, \theta_{2}\right), \\
\Psi^{(\alpha)}\left(\theta_{1}, \theta_{2}\right) \rightarrow e^{i f\left(\theta_{1}, \theta_{2}\right)} \Psi^{(\alpha)}\left(\theta_{1}, \theta_{2}\right) .
\end{gathered}
$$


Hence, the Chern number integral is conserved under this gauge transformation and it encapsulates general properties of the system. The Chern number has been used extensively in the quantum Hall literature for characterization of localized and extended states (Ref. [36], and references therein). In this paper, we use the Chern number as an indicator of order in the system. Moreover, it enables us to characterize the ground state in different regimes, especially where the calculation of the overlap with the Laughlin wave function fails to give a conclusive answer.

Before explaining the method for calculating the Chern number, we clarify some issues related to the degeneracy of the ground state. In some systems, the ground state can be degenerate; this can be intrinsic or it can be as a result of the topology of the system. If the ground state is degenerate, we should generalize the simple integral form of Eq. (12) to take into account the redundancy inside the ground-state manifold. For example, in the case of a twofold-degenerate ground state, there is an extra gauge freedom related to the relative phase between two ground states, and this freedom should be fixed. In other words, as we change the twist angles, we cannot keep track of the evolution of both states, since one cannot distinguish them from each other. Therefore, to uniquely determine the Chern number of the ground state(s), we should resolve this gauge invariance, which is treated in Secs. III B and III C.

It is important to note that the degeneracy in the noninteracting regime is fundamentally different from the degeneracy in the interacting case. In the noninteracting limit, the degeneracy can be lifted by a local perturbation-e.g., impurities - while in the hard-core case, the degeneracy remains in the thermodynamic limit [37]. The latter degeneracy in the ground state is a consequence of the global nontrivial properties of the manifold on which the particles move rather than related to a symmetry breaking which happens in conventional models - e.g., the Ising model. The topological degeneracy is not a consequence of breaking of any symmetry only in the ground state; instead, it is the entire Hilbert space which is split into disconnected pieces not related by any symmetry transformation. With a general argument, Wen [38] showed that if the degeneracy of a chiral spin system moving on a simple torus is $k$, then it should be $k^{g}$ on a torus with $g$ handles (Riemann surface with genus $g$ ); therefore, the topological degeneracy is an intrinsic feature of the system. In particular, in the context of the quantum Hall effect, this multicomponent feature of the ground state on a torus has a physical significance: while the single-component ground state on a sphere boundary condition gives zero conductance, the torus geometry with multicomponent ground state results in a correct conductance measured in the experiment, since the torus boundary condition is more relevant to the experiment. Changing twist angles of the boundary will rotate these components into each other and gives an overall nonzero conductance [34].

As studied in a recent work by Oshikawa and Senthil [39], as a universal feature, it has been shown that in the presence of a gap, there is a direct connection between the fractionalization and the topological order. More precisely, once a system has some quasiparticles with fractional statistics, a topological degeneracy should occur, which indicates the presence of a topological order. Therefore, the amount of degeneracy is related to the statistics of the fractionalized quasiparticles; e.g., in the case of $\nu=1 / 2$, the twofold degeneracy is related to $1 / 2$ anionic statistics of the corresponding quasiparticles. The Chern number has been also studied for spin-1/2 systems on a honeycomb lattice [40] for identifying Abelian and non-Abelian anions. Bellissard et al. [41] studied the Chern number for a disordered Fermi system using a noncommutative geometry.

To resolve the extra gauge freedom related to the two degenerate ground states, we consider two possibilities: (i) lifting the degeneracy by adding some impurities and (ii) fixing the relative phase between the two states in the ground state. Below, we explore both cases. In the first case, we introduce some fixed impurities to lift the degeneracy in the ground state for all values of the twist angles. This is an artifact of the finite size of the system which we take advantage of. In the presence of perturbations, we show that the system has a topological order in spite of the poor overlap with the Laughlin state. In the second approach, we use a scheme recently proposed by Hatsugai $[42,43]$ which is a generalized form for degenerate manifolds.

\section{B. Resolving the degeneracy by adding impurities}

In this section, we introduce some perturbations into the finite system in the form of local potentials (similar to local impurities in electronic systems) to split the degeneracy of the ground state and resolve the corresponding gauge invariance, which allows us to compute the Chern number. Furthermore, the fact that we can still uniquely determine the Chern number in the presence of impurities shows that the system retains its topological order, even when the impurities distort its ground-state wave function away from the Laughlin wave function.

In the context of the quantum Hall effect, the conventional numerical calculation of various physical quantities such as the energy spectrum, screening charge density profile, wave functions overlaps, and the density-density correlation functions cannot be used for understanding the transport properties of electrons in the presence of impurities (although useful for studying isolated impurities [44,45]). Recently, Sheng et al. [36] calculated the Chern number as an unambiguous way to distinguish insulating and currentcarrying states in the $\nu=1 / 3$ quantum Hall regime which correspond to zero and nonzero Chern numbers, respectively. In this work, a weak random disorder was used to lift the degeneracy of the ground state (threefold for $\nu=1 / 3$ ) for a finite number of electrons. The energy splitting between the lowest three states then decreased with increasing number of particles, which indicates the recovery of degeneracy in the thermodynamic limit. Moreover, the mobility gap can be determined by looking at the energy at which the Chern number drops toward zero. This energy gap is comparable to the energy gap obtained from the experiment, and it is not necessarily equal to the spectrum gap which separates the degenerate ground state from the excited states This shows the significance of Chern number calculations for understanding these systems. 
In a finite system, the coupling to a single-body interaction-e.g., impurities - can lift the degeneracy and one can uniquely determine the Chern number for the individual states by direct integration of Eq. (12). On the one hand, the impurity should be strong enough to split the energy in the ground state (in this case $E_{2}, E_{1}$, where $E_{j}$ denotes the energy of the $j$ th energy level) for all values of the twist angles. On the other hand, these impurities should be weak enough so that the energy splitting in the ground state remains smaller than the thermodynamic gap (in this case $E_{2}-E_{1} \ll E_{3}-E_{2}$ ).

To calculate the Chern number of an individual level, as mentioned in the previous section, we have to fix the phase of the wave function. The method that we explore in this section can be considered as a simplified version of the general method developed by Hatsugai [43] which we will explore in the next section. Following Kohmoto's procedure [46], we assume that the ground state $\Psi\left(\theta_{1}, \theta_{2}\right)$ may be expanded for all twist angles on an s-dimensional Hilbert discrete space $\Psi\left(\theta_{1}, \theta_{2}\right)=\left(c_{1}, c_{2}, \ldots, c_{s}\right)$. If $\mathcal{A}_{j}\left(\theta_{1}, \theta_{2}\right)$ in Eq. (12) is a periodic function on the torus of the boundary condition, then by application of Stokes' theorem, the Chern number will be always zero. The nontriviality (nonzero conductance in the case of the quantum Hall system) occurs because of the zeros of the wave function, where the phase is not well defined. Therefore, $\mathcal{A}\left(\theta_{1}, \theta_{2}\right)$ is not well defined everywhere and its flux integral can be nonzero. To uniquely determine the Chern number, we assume that the wave function and the vector field are not defined for certain points $\left(\theta_{1}^{n}, \theta_{2}^{n}\right)$ in $S_{n}$ regions on the torus of the boundary condition. For simplicity, we first discuss this procedure in the case of a nondegenerate ground state. For calculating the integral, we should acquire another gauge convention for the wave function inside these $S_{n}$ regions; e.g., in a discrete system, we may require an arbitrary element of the wave function to be always real, and thereby we can define a new vector field $\mathcal{A}_{j}^{\prime(\alpha)}\left(\theta_{1}, \theta_{2}\right)$, which is well defined inside these regions. These two vector fields differ from each other by a gauge transformation (Fig. 4):

$$
\mathcal{A}_{j}^{(\alpha)}\left(\theta_{1}, \theta_{2}\right)-\mathcal{A}_{j}^{\prime(\alpha)}\left(\theta_{1}, \theta_{2}\right)=\partial_{j} \chi\left(\theta_{1}, \theta_{2}\right),
$$

and the Chern number reduces to the winding number of the gauge transformation $\chi\left(\theta_{1}, \theta_{2}\right)$ over small loops encircling $\left(\theta_{1}^{n}, \theta_{2}^{n}\right)$, i.e., $\partial S_{n}$,

$$
C(\alpha)=\sum_{n} \frac{1}{2 \pi} \oint_{\partial S_{n}} \vec{\nabla} \chi \cdot d \vec{\theta}
$$

The one-dimensional gauge equation (15) should be resolved by making two conventions. For example, in one convention the first element and in the other the second element of the wave function in the Hilbert space should be reali.e., transforming the ground state $\Psi$ into $\Psi_{\Phi}=P \Phi=\Psi \Psi^{\dagger} \Phi$ where $\Phi=(1,0, \ldots, 0)^{\dagger}$ is an $\mathbf{s}$-dimensional vector and $P$ is a projection into the ground state and similarly with the other reference vector $\Phi^{\prime}=(0,1, \ldots, 0)^{\dagger}$. Since the gauge that relates two vector fields is the same as the one that relates the corresponding wave functions [similar to Eq. (15)], we can uniquely determine the gauge transformation function $\chi$ by evaluating $\Omega\left(\theta_{1}, \theta_{2}\right)=e^{i \chi}=\Phi^{\dagger} P \Phi^{\prime}$. Therefore, the Chern number will be equal to the number of windings of $\chi$ around regions where $\Lambda_{\phi}=\Phi^{\dagger} P \Phi=\left|c_{1}\right|^{2}$ is zero. Counting the vorticities has a vigorous computational advantage over the conventional method of direct integration of Eq. (12). In the direct integration, we need to choose a large number of mesh points for the boundary angles, because of the discrete approximation of derivatives in Eq. (12), and this makes the calculation computationally heavy. We note that for the system on a lattice, we should exactly diagonalize the Hamiltonian which is a sparse matrix as opposed to the continuum case where the Hamiltonian is a dense matrix residing on a smaller projected Hilbert space (lowest Landau level).

For removing the degeneracy, in our numerical simulations, we add a small amount of impurity which is modeled as $\delta$-function potentials located at random sites with a random strength of the order of the tunneling energy $J$. This is described by a Hamiltonian of the form $H=\Sigma_{i} U_{i}^{i m} \hat{n_{i}}$, where $i$ numerates the lattice site, $\hat{n}_{i}$ is the atom number operator, and $U_{i}^{i m}$ is the strength of the impurity at site $i$.

We choose reference states $\Phi$ and $\Phi^{\prime}$ to be eigenvectors of the numerically diagonalized Hamiltonian at two different twist angles. In Fig. 5, vorticities of $e^{i \chi}$ associated with the first and second energy levels is depicted. It is easy to see that the Chern number associated with the two ground states is 1 . The number of vortices may vary for the first and second ground states, but their sum is always equal to 1 . The hard-core limit $(U \gg J)$ is very similar to the case of the fractional quantum Hall effect, which, in the context of Hall systems, means a share of $1 / 2$ (in $e^{2} / h$ unit) for each ground state [35]. When the on-site interaction strength is small ( $U$ $<J$ ), the thermodynamic gap becomes comparable to the ground-state energy splitting $E_{2}-E_{1} \sim E_{3}-E_{2}$, the Chern number cannot be uniquely determined, and the system does not have topological order. On the other hand, in the limit of strong interaction $(U \gg J)$, the total Chern number associated with the ground states is equal to 1 , regardless of the impurity configuration. Moreover, in the hard-core limit, although the ground state is not described by the Laughlin wave function, since it is distorted by the impurity (in our model it can be as low as $50 \%$ ), the Chern number is unique and robust. This is an indication that the topological order is not related to the symmetries of the Hamiltonian and it is robust against arbitrary local perturbations [37]. These results indicate the existence of a topological order in the system and robustness of the ground states against local perturbations.

\section{Gauge fixing}

The method developed in the previous section has the graphical vortex representation for the Chern number which makes it computationally advantageous compared to the direct integration of Eq. (12). It cannot, however, be applied directly to a degenerate ground state, and therefore we had to introduce an impurities potential which lifted the degeneracy. On the other hand, a significant amount of impurities in the system may distort the energy spectrum, so that the underlying physical properties of the lattice and fluxes could be confounded by artifacts due to the impurities, especially for large $\alpha$. To address this issue, in this section, we explore a 

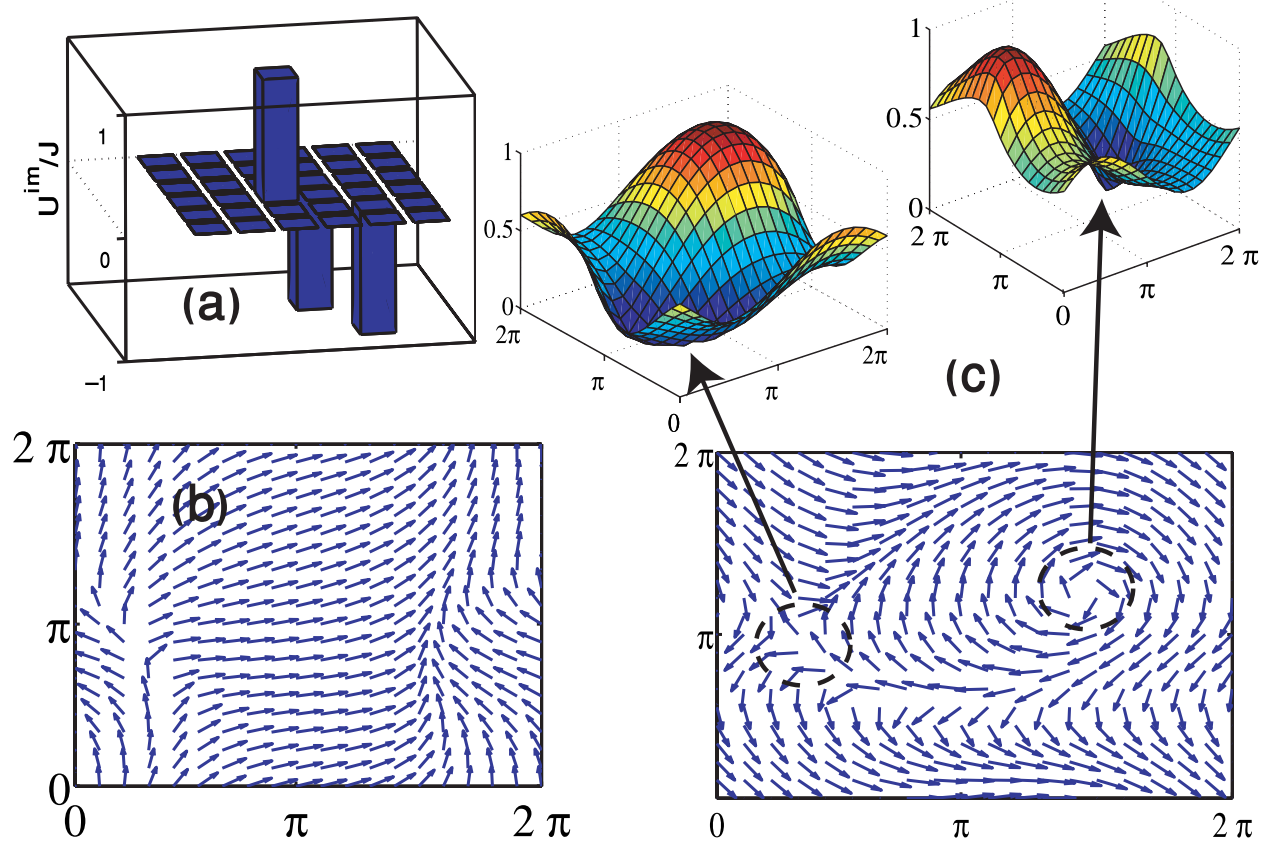

generalized method of the previous section based on Refs. $[42,43]$, which works for a degenerate ground-state.

By generalizing the Chern number formalism for a degenerate ground-state manifold, instead of having a single vector field $\mathcal{A}_{j}^{(\alpha)}\left(\theta_{1}, \theta_{2}\right)$, a tensor field $\mathcal{A}_{j}^{(\alpha, \beta)}\left(\theta_{1}, \theta_{2}\right)$ should be defined, where $\alpha, \beta=1,2, \ldots, q$ for a $\mathbf{q}$-fold degenerate ground state:

$$
\mathcal{A}_{j}^{(\alpha, \beta)}\left(\theta_{1}, \theta_{2}\right) \doteq i\left\langle\Psi^{(\alpha)}\left|\frac{\partial}{\partial \theta_{j}}\right| \Psi^{(\beta)}\right\rangle .
$$

Similar to the nondegenerate case, when $\mathcal{A}_{j}^{(\alpha, \beta)}$ is not defined, a new gauge convention should be acquired for the regions with singularities. This gives rise to a tensor gauge transformation $\chi^{(\alpha, \beta)}\left(\theta_{1}, \theta_{2}\right)$ on the border of these regions:

$$
\mathcal{A}_{j}^{(\alpha, \beta)}\left(\theta_{1}, \theta_{2}\right)-\mathcal{A}_{j}^{(\alpha, \beta)}\left(\theta_{1}, \theta_{2}\right)=\partial_{j} \chi^{(\alpha, \beta)}\left(\theta_{1}, \theta_{2}\right) .
$$

Following Hatsugai's proposal [43] for fixing the groundstate manifold gauge, we take two reference multiplets $\Phi$ and $\Phi^{\prime}$ which are two arbitrary $\mathbf{s} \times \mathbf{q}$ matrices; $q$ is the ground-state degeneracy (equal to 2 in our case). In our numerical simulation, we choose the multiplets to be two sets of ground states at two different twist angles far from each other-e.g., $(0,0)$ and $(\pi, \pi)$. We define an overlap matrix as $\Lambda_{\phi}=\Phi^{\dagger} P \Phi$, where $P=\Psi \Psi^{\dagger}$ is again the projection into the ground-state multiplet, and consider the regions where $\operatorname{det} \Lambda_{\Phi}$ or $\operatorname{det} \Lambda_{\Phi^{\prime}}$ vanishes (similar to zeros of the wave function in the nondegenerate case). Hence, the Chern number for $\mathbf{q}$ degenerate states will be equal to the total winding number of $\operatorname{Tr} \chi^{(\alpha, \beta)}$ for small neighborhoods $S_{n}$, in which $\operatorname{det} \Lambda_{\Phi}$ vanishes

$$
C(1,2, \ldots, q)=\sum_{n} \frac{1}{2 \pi} \oint_{\partial S_{n}} \vec{\nabla} \operatorname{Tr} \chi^{(\alpha, \beta)} d \vec{\theta}
$$

which is the same as the number of vortices of $\Omega\left(\Phi, \Phi^{\prime}\right)$ $=\operatorname{det}\left(\Phi^{\dagger} P \Phi^{\prime}\right)$. It should be noted that the zeros of $\operatorname{det} \Lambda_{\Phi}$
FIG. 5. (Color online) Chern number associated with low-lying energy states in the presence of impurities. Due to the impurity potential (a), the twofold-degenerate ground state splits and the wave function overlap with the Laughlin state drops to $52 \%$ and $65 \%$ for the first and second energy states, respectively. The results are for three atoms on a 6 $\times 6$ lattice $(\alpha=0.17)$ in the hardcore limit. (b) $\Omega\left(\theta_{1}, \theta_{2}\right)$ for the first level has no vorticity. However, for the second level, as shown in (c), $\Omega\left(\theta_{1}, \theta_{2}\right)$ has vorticity equal to 1 associated with regions where either $\Lambda_{\phi}$ or $\Lambda_{\phi}^{\prime}$ vanishes.

and $\operatorname{det} \Lambda_{\Phi}^{\prime}$ should not coincide in order to uniquely determine the total vorticity. In Fig. 6 , we plot $\Omega$, det $\Lambda_{\Phi}$, and $\operatorname{det} \Lambda_{\Phi^{\prime}}$, found by numerical diagonalization of the Hamiltonian for a mesh $(30 \times 30)$ of winding angles $\theta_{1}$ and $\theta_{2}$. In this figure, the Chern number can be determined be counting the number of vortices and it is readily seen that the winding number is equal to 1 for the corresponding zeros of $\operatorname{det} \Lambda_{\Phi}$ (or $\operatorname{det} \Lambda_{\Phi^{\prime}}$ ). Similarly, Fig. 7 shows vortices of $\Omega$ for a higher $\alpha$. Although the number of vortices increases in this case, the sum over signed vortices gives the same Chern number equal to 1 .

We have calculated the Chern number for fixed $\nu=1 / 2$ and different $\alpha$ 's by the approach described above. The result is shown in Table I. For low $\alpha \ll 1$, we know from Sec. II that the ground state is the Laughlin state and we expect to get a Chern number equal to 1 . For higher $\alpha$, the lattice structure becomes more apparent and the overlap with the Laughlin state decreases. However, in our calculation, the ground state remains twofold degenerate and it turns out that the groundstate Chern number tends to remain equal to 1 before reaching some critical $\alpha_{c} \simeq 0.4$. Hence, also in this regime we expect to have similar topological order and fractional statistics of the excitations above these states on the lattice.

For the arguments above to be applicable, it is essential that we can uniquely identify a twofold-degenerate ground state which is well separated from all higher-lying states. For higher flux densities $\alpha>\alpha_{c}$, the twofold ground-state degeneracy is no longer valid everywhere on the torus of the boundary condition. In this regime, the issue of degeneracy is more subtle, and the finite-size effect becomes significant. The translational symmetry argument [28], which was used in Sec. II, is not applicable on a lattice, and as pointed out by Kol and Read [47] the degeneracy of the ground state may vary periodically with the system size. Some of the gaps which appear in the calculation may be due to the finite size and vanish in the thermodynamic limit, whereas others may represent real energy gaps which are still present in the ther- 


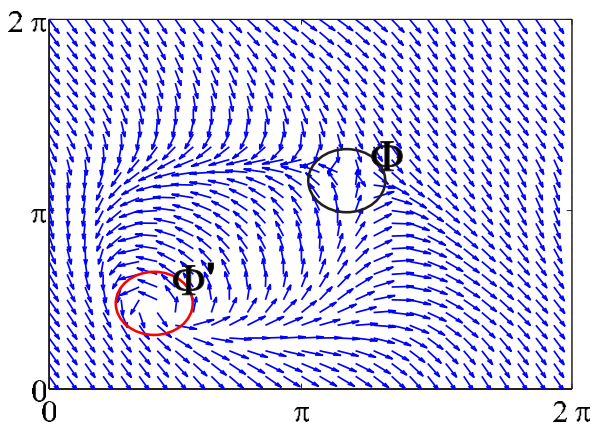

(a)

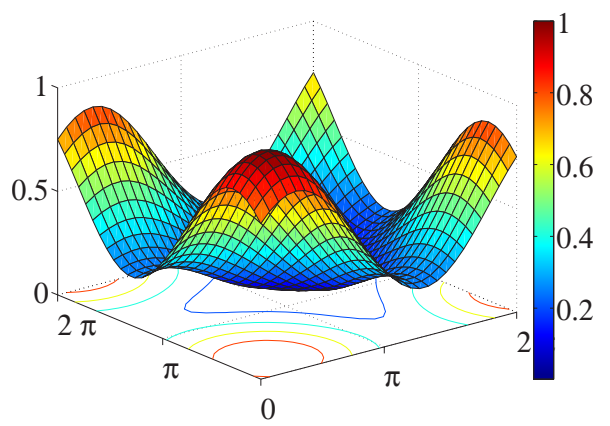

(b)

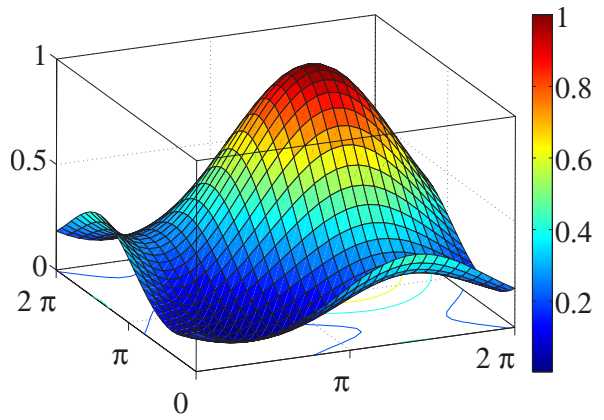

(c)

FIG. 6. (Color online) (a) shows the argument of $\Omega\left(\theta_{1}, \theta_{2}\right)$ as arrows for fixed $\Phi$ and $\Phi^{\prime}$. (b) and (c) Surface plots of det $\Lambda_{\Phi}$ and $\operatorname{det} \Lambda_{\Phi}^{\prime}$ (blue is lower than red). $\theta_{1}$ and $\theta_{2}$ change from zero to $2 \pi$. These plots have been produced for three atoms with $N_{\phi}=6(\alpha$ $=0.24)$ in the hard-core limit on a $5 \times 5$ lattice. The total vorticity corresponding to each of the reference wave functions $\left(\Phi\right.$ or $\left.\Phi^{\prime}\right)$ indicates a Chern number equal to 1 .

modynamic limit. To investigate this, we study the groundstate degeneracy as a function of boundary angles $\left(\theta_{1}, \theta_{2}\right)$ which are not physically observable and therefore the degeneracy in the thermodynamic limit should not depend on their value. In particular, Fig. 8 shows the energy levels of five particles at $\alpha=0.4$ for different values of the twist angles. The first and second levels are split at $\left(\theta_{1}=\theta_{2}=0\right)$, while they touch each other at $\left(\theta_{1}=\theta_{2}=\pi\right)$. We have observed similar behavior for different numbers of particles and lattice sizese.g., three and four atoms at $\alpha=0.5$. In this case, the system seems to not have a twofold degeneracy. Therefore, the ground state enters a different regime which is a subject for further investigation.

For having the topological order, it is not necessary to be in the hard-core limit. Even at finite interaction strength $U$

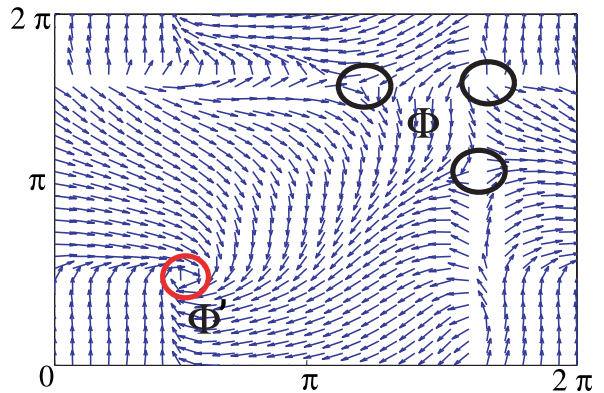

FIG. 7. (Color online) $\Omega\left(\theta_{1}, \theta_{2}\right)$ for fixed $\Phi$ and $\Phi^{\prime}$. $\theta_{1}$ and $\theta_{2}$ change from zero to $2 \pi$. This plot has been produced for four atoms with $N \phi=8$ in the hard-core limit on a $5 \times 5$ lattice $(\alpha=0.32)$. Although there are more vortices here compared to Fig. 6 , the total vorticity corresponding to each of the trial functions $\left(\Phi\right.$ or $\left.\Phi^{\prime}\right)$ indicates a Chern number equal to 1 .

$\sim J \alpha$, we have observed the same topological order with the help of the Chern number calculation. If $U$ gets even smaller, the energy gap above the ground state diminishes (as seen in Sec. II) and the topological order disappears.

We conclude that the Chern number can be unambiguously calculated for the ground state of the system in a regime where Laughlin's description is not appropriate for the lattice. The nonzero Chern number of a twofold-degenerate ground state, in this case equal to one-half per state, is a direct indication of the topological order of the system.

\section{EXTENSION OF THE MODEL}

In Secs. II and III above, we have investigated the conditions under which the fractional quantum Hall effect may be realized for particles on a lattice. The motivation for this study is the possibility to generate the quantum Hall effect with ultracold atoms in an optical lattice but the results of these sections are applicable regardless of the method used to attain this situation. In this and the following sections, we investigate some questions which are of particular relevance to ultracold atoms in an optical lattice. First, we introduce a long-range-e.g., dipole-dipole-interaction which turns out to increase the energy gap and thereby stabilizes the quantum

TABLE I. Chern number for different configurations in the hardcore limit for fixed filling factor $\nu=1 / 2$. The Laughlin state overlap is shown in the last column. Although the ground state deviates from the Laughlin state, the Chern number remains equal to onehalf per state before reaching some critical $\alpha_{c} \simeq 0.4$ where the energy gap vanishes.

\begin{tabular}{ccccc}
\hline \hline Atoms & Lattice & $\alpha$ & Chern/state & Overlap \\
\hline 3 & $6 \times 6$ & 0.17 & $1 / 2$ & 0.99 \\
4 & $6 \times 6$ & 0.22 & $1 / 2$ & 0.98 \\
3 & $5 \times 5$ & 0.24 & $1 / 2$ & 0.98 \\
3 & $4 \times 5$ & 0.3 & $1 / 2$ & 0.91 \\
4 & $5 \times 5$ & 0.32 & $1 / 2$ & 0.78 \\
3 & $4 \times 4$ & 0.375 & $1 / 2$ & 0.29 \\
\hline \hline
\end{tabular}



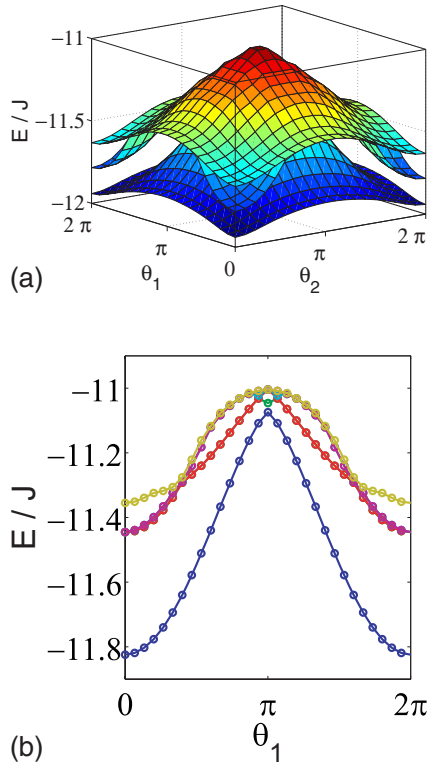

FIG. 8. (Color online) Low-lying energy levels as a function of twist angles. For high $\alpha$ the degeneracy of the ground state is a function of twist angles. The shown results are for five atoms on a $5 \times 5$ lattice; i.e., $\alpha=0.4$ (a) shows first three energy manifolds as a function of the toroidal boundary condition angles and (b) shows a cross section of (a) at $\theta_{2}=\pi$ for seven lowest-energy levels. The first and second energy levels get close to each other at $\theta_{1}=\theta_{2}=\pi$.

Hall states. We then turn to the case of $\nu=1 / 4$ and show that in order to realize this state, it is essential to have some kind of long-range interaction.

\section{A. Effect of the long-range interaction}

In an experimental realization of the quantum Hall effect on a lattice, it is desirable to have as large an energy gap as possible in order to be insensitive to external perturbations. So far, we have studied effect of the short-range interaction and we have shown that the gap increases with increasing interaction strength, but the value of the gap saturates when the short-range interaction becomes comparable to the tunneling energy $J$.

In this section, we explore the possibility of increasing the gap by adding a long-range repulsive dipole-dipole interaction to the system. Previously, such a dipole-dipole interaction was also studied in Ref. [9] as a method to achieve Read-Rezayi states [48] of rapidly rotating cold trapped atoms for $\nu=3 / 2$ and as a means to realize fractional quantum Hall physics with Fermi gases [49]. The dipole-dipole (magnetic or electric) interaction is described by the Hamiltonian

$$
H_{\mathrm{d}-\mathrm{d}}=U_{\text {dipole }} \sum_{1 \leqslant i<j \leqslant N} \frac{\mathbf{p}_{i} \cdot \mathbf{p}_{j}-3\left(\mathbf{n}_{i j} \cdot \mathbf{p}_{i}\right)\left(\mathbf{n}_{i j} \cdot \mathbf{p}_{j}\right)}{\left|\mathbf{r}_{i}-\mathbf{r}_{j}\right|^{3}},
$$

where $\mathbf{n}_{i j}=\left(\mathbf{r}_{i}-\mathbf{r}_{j}\right) /\left|\mathbf{r}_{i}-\mathbf{r}_{j}\right| \cdot \mathbf{p}_{i}$ are unit vectors representing the permanent dipole moments and the position vectors $\mathbf{r}_{i}$ are in units of the lattice spacing a. For simplicity, we assume that all dipoles are polarized in the direction perpendicular to the plane. With time-independent dipoles, the strength of the interaction is given by $U_{\text {dipole }}=\frac{\mu_{0} \mu^{2}}{4 \pi a^{3}}$ (or $\frac{\wp^{2}}{4 \pi \epsilon_{0} a^{3}}$ ) where $\boldsymbol{\mu}$ 's (ø’s) are the permanent magnetic- (electric-) dipole moment. Static dipoles will thus give the repulsive interaction $U_{\text {dipole }}$ $>0$, but experimentally time-varying fields may be introduced which effectively change the sign of the interaction [50]. For completeness, we shall therefore investigate both positive and negative $U_{\text {dipole }}$, but the repulsive interaction corresponding to static dipoles will be the most desirable situation since it stabilizes the quantum Hall states.

Experimentally the dipole-dipole interaction will naturally be present in the recently realized Bose-Einstein condensation of chromium [51] which has a large magnetic moment. However, for a lattice realization, polar molecules which have strong permanent electric-dipole moments are a more promising candidate. For typical polar molecules with the electric moment $\wp \sim 1 \mathrm{D}$, on a lattice with spacing a $\sim 0.5 \mu \mathrm{m}, U_{\text {dipole }}$ can be up to a few $\mathrm{kHz}$, an order of magnitude greater than the typical tunneling $J / 2 \pi \hbar$ which can be a few hundreds of $\mathrm{Hz}[1]$.

To study the effect of the dipole-dipole interaction, we again numerically diagonalize the Hamiltonian for a few hard-core bosons $(U \gg J)$, in the dilute regime $\alpha \leqslant 0.3$, while varying strength of the dipole-dipole interaction. The results of this simulation are shown in Fig. 9(a). After adding the dipole interaction, the ground state in a dilute lattice remains twofold degenerate, since the interaction only depends on the relative distance of the particles and keeps the center-of-mass motion intact. If the dipole interaction becomes too strong $\left(\mathbf{U}_{\text {dipole }} \gg J\right)$, the ground-state wave function deviates from the Laughlin wave function, but the topological order remains the same as for the system without a dipole interaction. We verified this by calculating the Chern number as explained in Sec. III for different values of the dipole-dipole interaction strength $\mathbf{U}_{\text {dipole }}$ and observed that the total Chern number of the twofold-degenerate ground state is equal to 1 . Moreover, as is shown in Fig. 9(b) adding such an interaction can increase the gap: the lower curve corresponds to the hard-core limit discussed in the previous work [10] and the upper curve corresponds to the system including the dipoledipole interaction. This enhancement varies linearly with the flux density $\alpha$ in a dilute lattice and does not depend on the number of particles and, consequently, it is expected to behave similarly in the thermodynamic limit.

One of the impediments of the experimental realization of the quantum Hall state is the smallness of the gap which can be improved by adding a dipole-dipole interaction. In this section, we showed that this improvement is possible and, moreover, by Chern number evaluation, we verified that adding dipole interaction does not change the topological behavior of the ground-state manifold.

\section{B. Case of $\nu=1 / 4$}

So far we have concentrated on the case of $\nu=1 / 2$. In this section, we briefly investigate the case of $\nu=1 / 4$. It is expected that the Laughlin wave function remains a good description for the ground state of a bosonic system for any even $q$, where $\nu=1 / q$. Following Haldane's argument [28], due to the center-of-mass motion, the degeneracy of the ground state is expected to be $q$-fold on a torus. Similar to 


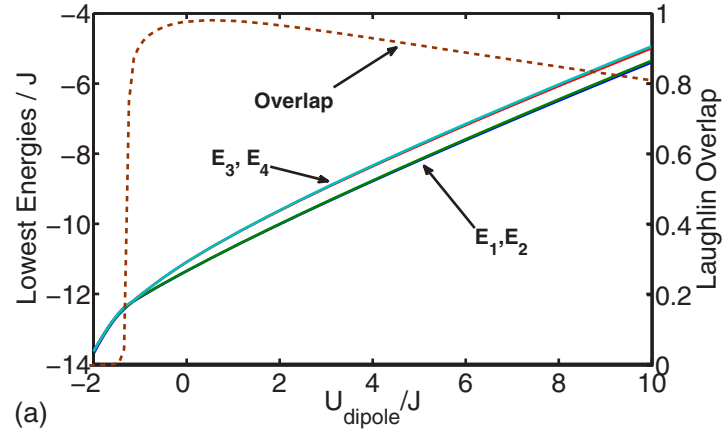

(a)

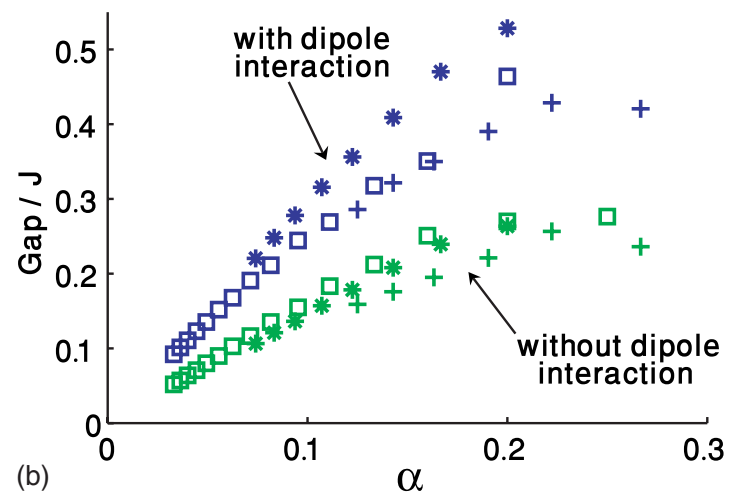

FIG. 9. (Color online) (a) The overlap of the ground state with the Laughlin wave function (dashed lines) and four low-lying energies of the system (solid lines) versus the dipole-dipole interaction for four atoms on a $6 \times 6$ lattice. (b) Gap enhancement for a fixed repulsive dipole-dipole interaction strength $U_{\text {dipole }}=5 \mathrm{~J}$ versus $\alpha$. The results are shown for $N=2(\square), N=3(*)$, and $N=4(+)$.

the case of $\nu=1 / 2$, the Laughlin wave function should be a suitable description for any $q$ provided that the magnetic field is weak so that we are close to the continuum limiti.e., $\alpha \ll 1$. Also the Chern number is expected to be equal to 1 for the $q$-fold degenerate ground state, which in the context of the quantum Hall effect means a share of $1 / q$ of the conduction quantum $e^{2} / h$ for each state in the $q$-fold degenerate ground-state manifold.

We have done both overlap and the Chern number calculations to check those premises. In the case of $\nu=1 / 4$, significant overlap occurs at low $\alpha \lesssim 0.1$. The average wavefunction overlap of four lowest-energy eigenstates with the Laughlin wave function is depicted in Fig. 10, where we have used a generalization of the Laughlin wave function for periodic boundary conditions similar to Eq. (4) [27].

We observe that the Laughlin wave function is a reliable description of the system with $\nu=1 / 4$ but only for much more dilute lattices $(\alpha \lesssim 0.1)$ compared to $\nu=1 / 2$ where significant overlap occurs for $\alpha \lesssim 0.3$. Contrary to $\nu=1 / 2$, where the gap is a fraction of the tunneling energy $J$, the gap for $\nu=1 / 4$ between the fourfold-degenerate ground state and the next energy level is infinitesimal. The reason for the vanishing gap can be understood in the continuum limit from the argument put forward in Ref. [52]: as noted previously the Laughlin wave function is an exact eigenstate of the Hamiltonian with an energy per particle equal to the lowest Landau level energy. The energy of the $m=4$ state is thus equal to the

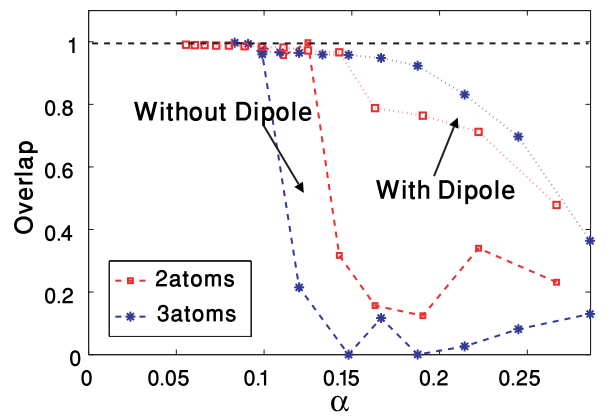

FIG. 10. (Color online) The overlap of the first four low-lying energy states with the Laughlin wave function for the case of $\nu$ $=1 / 4$ on a torus. The dashed (dotted) line shows the overlap for the system without (with) a dipole interaction $\left(U_{\text {dipole }}=5 J\right)$. The Laughlin state is only a good description for a more dilute lattice $\alpha$ $\lesssim 0.1$ compared to $\nu=1 / 2$. The dipole interaction stabilizes the system and the overlap is more significant for higher values of $\alpha$ $\lesssim 0.2$.

$m=2$ state. It thus costs a negligible energy to compress the $\nu=1 / 4$ state to the $\nu=1 / 2$ state, and therefore there is no gap. In an external trap the system will always choose the highest-density state which is the $\nu=1 / 2$ state. Note, however, that this argument only applies to short-range interactions. For long-range interactions, we expect to see a nonvanishing gap.

Even though that, with short-range interactions, the gap is very small in our numerical calculations, it is still sufficiently large that it allows us to unambiguously determine the Chern number for the ground-state manifold as described in Sec. III C. As expected the calculation shows that the Chern number is equal to 1 corresponding to a fourfold-degenerate ground state consistent with the generalization of the fermionic case in the fractional quantum Hall theory [34,35]. In Fig. 10, the overlap of the first four lowest-energy states with the Laughlin wave function is depicted. In the absence of the dipole interaction, the ground-state overlap is significant only for $\alpha \lesssim 0.1$; however, by adding a moderate dipole interaction $\left(U_{\text {dipole }}=5 J\right)$, the overlap becomes more significant for a larger interval of the flux density-i.e., $\alpha \lesssim 0.25$. This is due to the fact that states with lower density become more favorable in the presence of a long-range repulsive interaction.

We observed that adding a dipole interaction would lead to an improvement of the gap for $\nu=1 / 2$ and make the Laughlin overlap more significant for a larger interval of the magnetic field strength $\alpha$ in the case of $\nu=1 / 4$. Therefore this long-range interaction can be used as a tool for stabilizing the ground state and makes the realization of these quantum states experimentally more feasible.

\section{DETECTION OF THE QUANTUM HALL STATE}

In an experimental realization of the quantum Hall states, it is essential to have an experimental probe which can verify that the desired states were produced. In most experiments with cold-trapped atoms, the state of the system is probed by releasing the atoms from the trap and imaging the momen- 
tum distribution. In Ref. [10], it was shown that this technique provides some indication of the dynamics in the system. This measurement technique, however, only provides limited information, since it only measures the singleparticle density matrix and provides no information about the correlations between the particles. In Refs. [52,53] more advanced measurement techniques were proposed, where the particle correlation is obtained by looking at the correlations in the expansion images. In this section, we study Bragg scattering as an alternative measurement strategy which reveals the excitation spectrum of the quantum system. In Ref. [54] bosonic quantum Hall system responses to a perturbative potential are studied. We focus on Bragg scattering where two momentum states of the same internal ground state are connected by a stimulated two-photon process [55]. By setting up two laser beams with frequencies $\omega_{1}$ and $\omega_{2}$ and wave vectors $\vec{k}_{1}$ and $\vec{k}_{2}$ in the plane of the original lattice, a running optical superlattice will be produced with frequency $\omega_{1}-\omega_{2}$ and wave vector $\vec{k}_{1}-\vec{k}_{2}$. (Both frequencies $\omega_{1}$ and $\omega_{2}$ should be close to an internal electronic dipole transition in the atoms.) The beams should be weak and sufficiently detuned so that direct photon transitions are negligible-i.e., $\mathcal{E}_{1}, \mathcal{E}_{2}, \gamma \ll \omega_{1}-\omega_{0}, \omega_{2}-\omega_{0}$, where $\omega_{0}$ is the frequency of the transition, $\gamma$ is the corresponding spontaneous decay rate, and $\mathcal{E}_{1}, \mathcal{E}_{2}$ are the Rabi frequencies related to the laser-atom coupling. In this perturbative regime, the inelastic scattering of photons will be suppressed; the atom starts from the internal ground state, absorbs one photon from, e.g., beam 1 by going to a virtual excited state, and then emits another photon into beam 2 by returning to its internal ground state. After this process, the system has acquired an energy equal to $\hbar \omega=\hbar\left(\omega_{1}-\omega_{2}\right)$ and a momentum kick equal to $\vec{q}=\vec{k}_{1}-\vec{k}_{2}$. Therefore, the overall effect of the recoil process is a moving ac Stark shift as a perturbing moving potential, and the effective Hamiltonian represents the exchange of the two-photon recoil momentum and the energy difference to the system and is proportional to the local density-i.e., $H \propto \rho(r) e^{-i\left(\omega_{1}-\omega_{2}\right) t+i\left(\vec{k}_{1}-\vec{k}_{2}\right) \cdot \vec{r}}+$ c.c.

This process can be used to probe density fluctuations of the system and thus to measure directly the dynamic structure factor $S(q, \omega)$ and static structure factor $S(q)$. This kind of spectroscopy has been studied for a BEC in a trap by Blakie et al. [56] and Zambelli et al. [57] and has been realized experimentally in Refs. [58-63].

Also, Bragg spectroscopy in an optical lattice is discussed in Ref. [64] in a mean-field approach and also in Ref. [65] as a probe of the Mott-insulator excitation spectrum. On the other hand, in the context of quantum Hall effect, the static structure factor has been studied for probing the magnetron excitations [66] and charge density waves [67]. The dynamic and static structure factors are given, respectively, as

$$
\begin{gathered}
S(\vec{q}, \omega)=\sum_{n, 0}\left|\left\langle n\left|\rho^{\dagger}(\vec{q})\right| 0\right\rangle\right|^{2} \delta\left(\omega-E_{n}+E_{0}\right), \\
S(\vec{q})=\sum_{n, 0}\left|\left\langle n\left|\rho^{\dagger}(\vec{q})\right| 0\right\rangle\right|^{2}=\sum_{n, 0} \mid\langle n| \sum_{\overrightarrow{r_{i}}} e^{\left.i \vec{q} \cdot \overrightarrow{r_{i}}|0\rangle\right|^{2},}
\end{gathered}
$$

where the density fluctuation operator is defined as $\rho^{\dagger}(\vec{q})$ $=\sum_{m, n} \mathcal{A}_{\vec{q}}(m, n) c_{m}^{\dagger} c_{n}$ and the coefficients are defined as Fou-
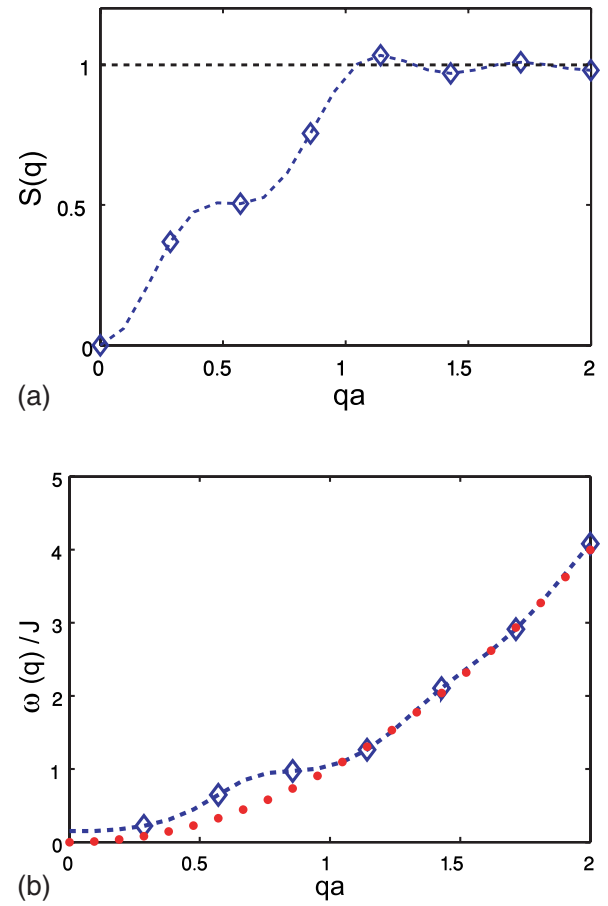

FIG. 11. (Color online) (a) Structure factor and (b) energy spectrum for a $11 \times 11$ lattice with three atoms on a torus. Points show the momentums allowed by the boundary conditions. The dotted line in (b) shows for comparison the low-energy spectrum of a free particle which equals $J(q a)^{2}$.

rier transforms of the Wannier functions: $\mathcal{A}_{\vec{q}}(m, n)$ $=\int d^{2} \vec{r} e^{i q \cdot \vec{r}} \phi^{*}\left(\vec{r}-\vec{r}_{m}\right) \phi\left(\vec{r}-\vec{r}_{n}\right)$, where the Wannier function $\phi\left(\vec{r}-\vec{r}_{n}\right)$ is the wave function of an atom localized on a site centered at $\vec{r}_{n}$. Below, we focus on deep optical lattices, where $\mathcal{A}_{\vec{q}}(m, n)=e^{i \vec{q} \cdot \vec{r}_{m}} \delta_{m, n}$.

In the structure factor, there is a sum over the excited states $|n\rangle$ and ground states $|0\rangle$ and the self-term is thus excluded. The ground state on a torus is twofold degenerate, and therefore in our numerics, we add the contribution of both.

Since we are working on a discrete lattice, there will be a cutoff in the allowed momentum given by the lattice spacing $q_{\max }=\pi / a$, where $a$ is the distance between lattice sites. Figure 11(a) shows the structure factor for the case $\nu=1 / 2$ for a small $\alpha$ calculated from our numerical diagonalizations. In the data presented here, we have chosen $\vec{q}=q \hat{x}$ and the result should be similar in other directions in the lattice plane. We see that $S(q)$ is modulated at a momentum corresponding to the magnetic length. For the parameters that we have investigated, the general features of the structure factor are independent of the size of the system.

We obtain the excitation spectrum shown in Fig. 11(b) similar to Ref. [66] by the Feynman-Bijl approximation. In the continuum limit $(\alpha \ll 1)$, we assume that the low-lying excitations are long-wavelength density oscillations and their wave functions can be approximated to have the form $\propto \rho_{k}|0\rangle$. Therefore, the variational estimate for the excitation energy is $\omega(q) \simeq \hbar^{2} q^{2} / 2 m S(q)$. At zero momentum and at the momentum corresponding to the magnetic length, there are gaps, and we also observe a deviation from the free-particle 
spectrum similar to the magnetron case as reminiscent of the Wigner crystal. It should be noted that the deviation does not depend on the size or the number of particles in the system. As clearly seen in Fig. 11 the energy spectrum and structure factor deviate from those of free particles; therefore, it could be used as an experimental probe of the system.

The structure factor and excitation spectrum imply some general features that are very different from that of the Mottinsulator and superfluid states, and can be used a powerful experimental indication of the quantum Hall states.

\section{GENERATING MAGNETIC HAMILTONIAN FOR NEUTRAL ATOMS ON A LATTICE}

Recently, there have been several proposals for producing artificial magnetic field for neutral atoms in optical lattices $[10,21,22]$, however, the implementation of each of them is still experimentally demanding. Recently, there has been an experimental demonstration of a rotating optical lattice [23] which is equivalent to an effective magnetic field (see below). The technique used in this experiment, however, generates a lattice with a large lattice spacing, because it uses laser beams which are not exactly counterpropagating. This longer spacing reduces the energy scale in the lattice and thereby also reduces quantities such as energy gaps. Here, we shall now introduce an alternative method for generating a rotating optical lattice, which does not result in an increased lattice spacing. This method consists of rotating the optical lattice by manipulating laser beams.

In a frame of reference rotating with angular velocity $\omega$ around the $z$ axis, the Hamiltonian for a particle of mass $m$ in a (planar) harmonic trap of natural frequency $\omega_{0}$ is

$$
\begin{aligned}
H & =\frac{p^{2}}{2 m}+\frac{1}{2} m \omega_{0}\left(x^{2}+y^{2}\right)-\omega \hat{z} \cdot(\vec{r} \times \vec{p}) \\
& =\frac{(p-m \omega \hat{z} \times \vec{r})^{2}}{2 m}+\frac{1}{2} m\left(\omega_{0}^{2}-\omega^{2}\right)\left(x^{2}+y^{2}\right) .
\end{aligned}
$$

At resonance $\omega_{0}=\omega$ the form is equivalent to the Hamiltonian of a particle of charge $q$ experiencing an effective magnetic field $B=\vec{\nabla} \times(m \omega \hat{z} \times \vec{r} / q)=(2 m \omega / q) \hat{z}$. Therefore, by simply rotating the optical lattice, we can mimic the magnetic field for neutral atoms.

To rotate the optical lattice, we propose to set up four acousto-optic modulators (AOMs) and four focusing composite lenses as shown in Fig. 12. By sweeping the acoustic wave frequency, the beams can be focused and make a rotating optical lattice.

In an AOM, for the first-order diffracted light we have $\sin \theta_{B}=\frac{\lambda}{2 \Lambda}$, where $\Lambda$ is the wavelength of sound in the medium, $\lambda$ is the light wavelength, and $\theta_{B}$ is half of the angle between a diffracted beam and the nondiffracted beam (Fig. 12). By increasing the frequency of the acoustic wave, the diffraction angle increases. However, the beam should be focused by a large-aperture lens so that it always passes the region where we want to make the optical lattice. By focusing a similar but counterpropagating beam, we can make a rotating standing wave (Fig. 12). By repeating the same con-

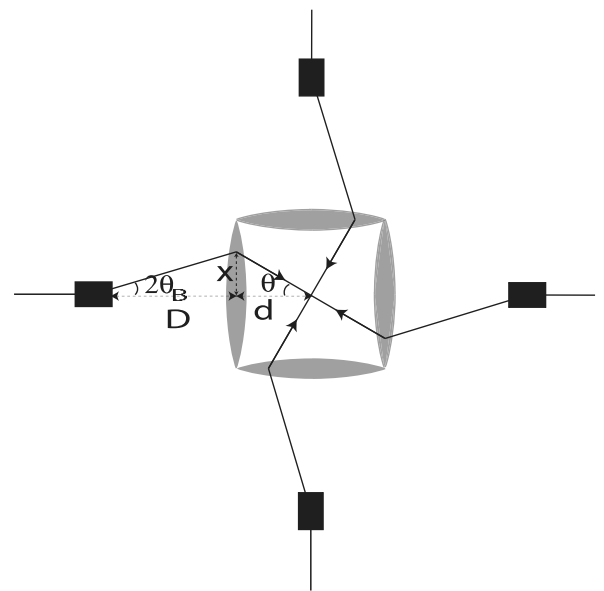

FIG. 12. Proposal for realizing a rotating optical lattice. Four AOMs (black boxes) change the direction of the lattice beams, which are subsequently focused in the middle of the setup by four lenses (gray). Simultaneously varying the four diffraction angles in the AOMs will generate a rotating optical lattice.

figuration in the transverse direction, we can make a rotating optical lattice. In particular, if the AOM is far from the composite lenses, $D \gg d$, then $x / D=\lambda / \Lambda$ and $x / d=\tan \theta$ where $-\pi / 4 \leqslant(\theta=\omega t) \leqslant \pi / 4$, where the parameters are defined in Fig. 12. If we consider a square lattice with dimensions $N_{x}$ $=N_{y}=\mathcal{N}$, the number of magnetic flux given by rotation is

$$
N_{\phi}=\frac{B A}{\Phi_{0}}=\frac{\pi}{2} \frac{\mathcal{N}^{2} \omega}{\omega_{r}},
$$

where $\omega_{r}=\hbar k^{2} / 2 M$ is the atomic recoil frequency.

On the other hand, the upper limit for the magnetic field comes from the single-Bloch-band approximation which we made in writing the Hamiltonian for the optical lattice. In order for the particles to remain in the first band, the traveling lattice beams should move them adiabatically. From Ref. [68], the adiabaticity condition for a moving lattice with an acceleration $\eta$ equal to $\omega^{2} \mathcal{N} \lambda / 4$ at the edge is $m \eta \lambda \ll \hbar \frac{\omega_{p}^{4}}{\omega_{r}^{3}}$, where $\omega_{p}$ is the frequency difference between the first and second bands in the lattice. This puts a limit on how large the lattice can become, $\mathcal{N} \ll \frac{\omega_{p}^{4}}{\omega^{2} \omega_{r}^{2}}$.

Hence, for $\nu=1 / 2$ with a lattice filling fraction $\frac{N}{\mathcal{N}^{2}} \sim \frac{1}{8}$ and a typical recoil frequency $\omega_{r}=(2 \pi) 4 \mathrm{kHz}$, one can enter the regime of fractional quantum Hall effect by rotating the lattice at $\omega \sim(2 \pi) 650 \mathrm{~Hz}$. If a deep optical lattice is usede.g., $\omega_{p} \sim 10 \omega_{r}$ - the adiabaticity condition is easily satisfied for a lattice of size $\mathcal{N} \sim 1000$. The experimentally challenging part will, however, likely be to mitigate the instability of the lattice caused by the thickness of the beam and aberration of the lenses at the turning points-i.e., near $\theta=\pi / 4$.

\section{CONCLUSIONS}

An extended study of the realization of the fractional quantum Hall states in optical lattices has been presented. We showed that a Hamiltonian similar to that of a magnetic field for charged particles can be constructed for neutral ul- 
tracold atoms and molecules confined in an optical lattice. By adding an on-site interaction for the case of $\nu=1 / 2$, an energy gap develops between the ground state and the first excited state, which increases linearly as $\alpha U$ and saturates to its value in the hard core limit $U \gg J$. We learned that the Laughlin wave function is a reliable description of the system for low flux densities $\alpha \lesssim 0.25$. However, for higher $\alpha$ 's, the lattice structure becomes more pronounced and a better description of the system can be carried out by investigating the Chern number associated with the ground-state manifold. The Chern number indicates that the system has topological order up to some critical flux density $\alpha_{c} \simeq 0.4$, where the properties of the ground-state manifold start to change. We have also studied $\nu=1 / 4$, where compared to $\nu=1 / 2$, the Laughlin wave function only describes the ground state for lower values of the flux $\alpha \leq 0.1$. We showed that a dipoledipole interaction can enhance the gap and stabilize the system, and therefore make the ground state more experimen- tally realizable. Bragg spectroscopy has been studied as a potential experimental tool to diagnose theses incompressible states.

Characterization of the ground state by evaluating the Chern number, developed in Sec. III C, can be generalized to other interesting many-body systems where the conventional overlap calculation fails to work. In particular, this method can be applied to ground states with non-Abelian statistics which are appealing candidates for fault-tolerant quantum computations.

\section{ACKNOWLEDGMENTS}

We thank K. Yang, M. Greiner, S. Girvin, and J. I. Cirac for fruitful discussions. This work was partially supported by the NSF Career Award, the Packard Foundation, AFSOR, and the Danish Natural Science Research Council.
[1] M. Greiner, O. Mandel, T. Esslinger, T. W. Hansch, and I. Bloch, Nature (London) 415, 39 (2002).

[2] O. Mandel, M. Greiner, A. Widera, T. Rom, T. W. Hansch, and I. Bloch, Nature (London) 425, 937 (2003).

[3] G. Campbell, J. Mun, M. Boyd, E. Streed, W. Ketterle, and D. Pritchard, Phys. Rev. Lett. 96, 020406 (2006).

[4] K. Winkler et al., Nature (London) 441, 853 (2006).

[5] S. Folling et al., Nature (London) 434, 481 (2005).

[6] J. R. Abo-Shaeer et al., Science 292, 476 (2001).

[7] N. K. Wilkin and J. M. F. Gunn, Phys. Rev. Lett. 84, 6 (2000).

[8] N. R. Cooper, N. K. Wilkin, and J. M. F. Gunn, Phys. Rev. Lett. 87, 120405 (2001).

[9] E. H. Rezayi, N. Read, and N. R. Cooper, Phys. Rev. Lett. 95, 160404 (2005).

[10] A. S. Sørensen, E. Demler, and M. D. Lukin, Phys. Rev. Lett. 94, 086803 (2005).

[11] A. Y. Kitaev, Ann. Phys. (N.Y.) 303, 2 (2003).

[12] F. E. Camino, W. Zhou, and V. J. Goldman, Phys. Rev. Lett. 95, 246802 (2005).

[13] F. E. Camino, W. Zhou, and V. J. Goldman, Phys. Rev. B 74, 115301 (2005).

[14] B. Paredes, P. Fedichev, J. I. Cirac, and P. Zoller, Phys. Rev. Lett. 87, 010402 (2001).

[15] D. Hofstadter, Phys. Rev. B 14, 2239 (1976).

[16] R. B. Laughlin, Phys. Rev. Lett. 50, 1395 (1983).

[17] D. Jaksch, C. Bruder, J. I. Cirac, C. W. Gardiner, and P. Zoller, Phys. Rev. Lett. 81, 3108 (1998).

[18] R. E. Peierls, Z. Phys. 80, 763 (1933).

[19] V. Bretin, S. Stock, Y. Seurin, and J. Dalibard, Phys. Rev. Lett. 92, 050403 (2004).

[20] V. Schweikhard, I. Coddington, P. Engels, V. P. Mogendorff, and E. A. Cornell, Phys. Rev. Lett. 92, 040404 (2004).

[21] D. Jaksch and P. Zoller, New J. Phys. 5, 56 (2003).

[22] E. J. Mueller, Phys. Rev. A 70, 041603(R) (2004).

[23] S. Tung, V. Schweikhard, and E. A. Cornell, Phys. Rev. Lett. 97, 240402 (2006).

[24] M. Popp, B. Paredes, and J. I. Cirac, Phys. Rev. A 70, 053612
(2004).

[25] E. A. Donley, N. R. Claussen, S. T. Thompson, and C. E. Wieman, Nature (London) 417, 529 (2002).

[26] S. Dürr, T. Volz, A. Marte, and G. Rempe, Phys. Rev. Lett. 92, 020406 (2004).

[27] N. Read and E. Rezayi, Phys. Rev. B 54, 16864 (1996).

[28] F. D. M. Haldane, Phys. Rev. Lett. 55, 2095 (1985).

[29] F. D. M. Haldane and E. H. Rezayi, Phys. Rev. Lett. 54, 237 (1985).

[30] E. Fradkin, Field Theories of Condensed Matter Systems (Addison-Wesley, Reading, MA, 1991).

[31] J. Gerton, M. Strekalov, I. Prodan, and R. Hulet, Nature (London) 408, 692 (2000).

[32] J. E. Avron, R. Seiler, and B. Simon, Phys. Rev. Lett. 51, 51 (1983).

[33] D. J. Thouless, M. Kohmoto, M. P. Nightingale, and M. den Nijs, Phys. Rev. Lett. 49, 405 (1982).

[34] Q. Niu, D. J. Thouless, and Y. S. Wu, Phys. Rev. B 31, 3372 (1985).

[35] R. Tao and F. D. M. Haldane, Phys. Rev. B 33, 3844 (1986).

[36] D. N. Sheng, X. Wan, E. H. Rezayi, K. Yang, R. N. Bhatt, and F. D. M. Haldane, Phys. Rev. Lett. 90, 256802 (2003).

[37] X. G. Wen and Q. Niu, Phys. Rev. B 41, 9377 (1990).

[38] X. G. Wen, Phys. Rev. B 40, 7387 (1990).

[39] M. Oshikawa and T. Senthil, Phys. Rev. Lett. 96, 060601 (2006).

[40] A. Kitaev, Ann. Phys. (N.Y.) 321, 2 (2005).

[41] J. Bellissard, A. van Elst, and H. Schulz-Baldes, J. Math. Phys. 35, 5373 (1994).

[42] R. Varnhagen, Nucl. Phys. B 443, 501 (1995).

[43] Y. Hatsugai, J. Phys. Soc. Jpn. 74, 1374 (2005).

[44] E. H. Rezayi and F. D. M. Haldane, Phys. Rev. B 32, 6924 (1985).

[45] F. C. Zhang, V. Z. Vulovic, Y. Guo, and S. Das Sarma, Phys. Rev. B 32, 6920 (1985).

[46] M. Kohmoto, Ann. Phys. (N.Y.) 160, 343 (1985).

[47] A. Kol and N. Read, Phys. Rev. B 48, 8890 (1993). 
[48] N. Read and E. Rezayi, Phys. Rev. B 59, 8084 (1999).

[49] M. A. Baranov, K. Osterloh, and M. Lewenstein, Phys. Rev. Lett. 94, 070404 (2005).

[50] S. Giovanazzi, A. Görlitz, and T. Pfau, Phys. Rev. Lett. 89, 130401 (2002).

[51] A. Griesmaier, J. Werner, S. Hensler, J. Stuhler, and T. Pfau, Phys. Rev. Lett. 94, 160401 (2005).

[52] R. N. Palmer and D. Jaksch, Phys. Rev. Lett. 96, 180407 (2006).

[53] E. Altman, E. Demler, and M. D. Lukin, Phys. Rev. A 70, 013603 (2004).

[54] R. Bhat et al., e-print arXiv:cond-mat/07053341.

[55] J. Stenger, S. Inouye, A. P. Chikkatur, D. M. Stamper-Kurn, D. E. Pritchard, and W. Ketterle, Phys. Rev. Lett. 82, 4569 (1999).

[56] P. B. Blakie, R. J. Ballagh, and C. W. Gardiner, Phys. Rev. A 65, 033602 (2000).

[57] F. Zambelli, L. Pitaevskii, D. M. Stamper-Kurn, and S. Stringari, Phys. Rev. A 61, 063608 (2000).

[58] D. M. Stamper-Kurn, A. P. Chikkatur, A. Görlitz, S. Inouye, S. Gupta, D. E. Pritchard, and W. Ketterle, Phys. Rev. Lett. 83,
2876 (1999).

[59] J. M. Vogels, K. Xu, C. Raman, J. R. Abo-Shaeer, and W. Ketterle, Phys. Rev. Lett. 88, 060402 (2002).

[60] R. Ozeri, J. Steinhauer, N. Katz, and N. Davidson, Phys. Rev. Lett. 88, 220401 (2002).

[61] J. Steinhauer, N. Katz, R. Ozeri, N. Davidson, C. Tozzo, and F. Dalfovo, Phys. Rev. Lett. 90, 060404 (2003).

[62] N. Katz, R. Ozeri, J. Steinhauer, N. Davidson, C. Tozzo, and F. Dalfovo, Phys. Rev. Lett. 93, 220403 (2004).

[63] S. R. Muniz, D. S. Naik, and C. Raman, Phys. Rev. A 73, 041605(R) (2006).

[64] C. Menotti, M. Kramer, L. Pitaevskii, and S. Stringari, Phys. Rev. A 67, 053609 (2003).

[65] A. M. Rey, P. B. Blakie, G. Pupillo, C. J. Williams, and C. W. Clark, Phys. Rev. A 72, 023407 (2005).

[66] S. M. Girvin, A. H. MacDonald, and P. M. Platzman, Phys. Rev. B 33, 2481 (1986).

[67] E. H. Rezayi, F. D. M. Haldane, and K. Yang, Phys. Rev. Lett. 83, 1219 (1999).

[68] M. Ben Dahan, E. Peik, J. Reichel, Y. Castin, and C. Salomon, Phys. Rev. Lett. 76, 4508 (1996). 